\title{
DSMC modeling of rarefied ionization reactions and applications to hypervelocity spacecraft reentry flows
}

\author{
Ming Fang ${ }^{1}$, Zhi-Hui Li $\mathrm{Li}^{1,2^{*}} \mathbb{D}$, Zhong-Hua Li ${ }^{1}$, Jie Liang ${ }^{1}$ and Yong-Hao Zhang ${ }^{3}$
}

\footnotetext{
* Correspondence: zhli0097@×263. net

${ }^{1}$ Hypervelocity Aerodynamics Institute, China Aerodynamics Research and Development Center, P.O.Box 211, Mianyang 621000, China

${ }^{2}$ National Laboratory for Computational Fluid Dynamics, BUAA, No.37 Xueyuan Road, Beijing 100191, China

Full list of author information is available at the end of the article
}

\begin{abstract}
The DSMC modeling is developed to simulate three-dimensional (3D) rarefied ionization flows and numerically forecast the communication blackout around spacecraft during hypervelocity reentry. A new weighting factor scheme for rare species is introduced, whose key point is to modify the corresponding chemical reaction coefficients involving electrons, meanwhile reproduce the rare species in resultants and preserve/delete common species in reactants according to the weighting factors. The resulting DSMC method is highly efficient in simulating weakly inhomogeneous flows including the Couette shear flow and controlling statistical fluctuation with high resolution. The accurate reliability of the present DSMC modeling is also validated by the comparison with a series of experimental measurements of the Shenzhou reentry capsule tested in a low-density wind tunnel from the HAI of CARDC. The obtained electron number density distribution for the RAM-C II vehicle agrees well with the flight experiment data, while the electron density contours for the Stardust hypervelocity reentry match the reference data completely. In addition, the present 3D DSMC algorithm can capture distribution of the electron, $\mathrm{N}^{+}$and $\mathrm{O}^{+}$number densities better than the axis-symmetric DSMC model. The introduction of rare species weighting factor scheme can significantly improve the smoothness of the number density contours of rare species, especially for that of electron in weak ionization case, while it has negligible effect on the macroscopic flow parameters. The ionization characteristics of the Chinese lunar capsule reentry process are numerically analyzed and forecasted in the rarefied transitional flow regime at the flying altitudes between 80 and $97 \mathrm{~km}$, and the simulations predict communication blackout altitudes which are in good agreement with the actual reentry flight data. For the spacecraft reentry with hypervelocity larger than the second cosmic speed, it is forecasted and verified by the present DSMC modeling that ionization reactions will cover the windward capsule surface, leading to reentry communication blackout, and the communication interruption must be considered in the communication design during reentry in rarefied flow regimes.
\end{abstract}

Keywords: Spacecraft hypervelocity reentry, Chemical reaction, Air ionization effect, Rarefied gas dynamics, DSMC method, Communication blackout 


\section{Introduction}

With rapid development of manned spaceflight, lunar and deep space exploration, recyclable spacecraft reentry speed has been increasing from the first to the second cosmic velocity, even higher. Therefore, typical hypervelocity flow phenomena such as thermo-chemical nonequilibrium, ionization and communication blackout occur also in rarefied flows [1, 2]. For instance, the Chinese lunar exploration capsule launched in 2014 returned with a speed of $11.2 \mathrm{~km} / \mathrm{s}$, while the Stardust recorded the fastest man-made spacecraft reentry speed of $12.8 \mathrm{~km} / \mathrm{s}$ [3-5]. Under these typical hypersonic conditions, the extremely strong bow shock wave around the vehicle leads to intensive physico-chemical nonequilibrium process, thus the atmosphere needs to be considered as an ionized gas mixture with vibrational excitation, dissociation and electronic excitation. To improve communication design of spacecraft during hypervelocity reentry, we need to accurately predict the electron density distribution at the complex three-dimensional (3D) surface of spacecraft, which has been a long-standing research challenge $[6,7]$ of the extreme flows in the field of fluid mechanics. At low altitude, the ionization effect is usually treated as the conventional chemical reaction using a continuum flow model such as the NavierStokes equation [8, 9]. However, the continuum flow assumption for the NavierStokes equation becomes invalid for rarefied gas at high altitude. Ground test facilities can hardly reproduce the crucial non-equilibrium flows of extremely highspeed reentry in non-continuum atmospheric environment [10]. Although kinetic methods including the direct solver of kinetic models [11-15], the discrete velocity models [16-22], the gas-kinetic unified algorithm [23-27], the unified flow solver $[28,29]$, and the BGK-type discrete unified gas-kinetic schemes [30, 31] can describe non-equilibrium gas flows, they are still under development and computationally expensive for hypersonic flows. Currently, the most widely used method for rarefied hypersonic flows is the Direct Simulation Monte Carlo (DSMC) [32-34] method. In 1987, Bird [35] utilized the DSMC method to simulate the rarefied air ionization for the first time, where the ionization process was treated using 11 molecular species and 41 chemical reactions, and the electrons in close proximity to the ions were kept in order to enforce charge neutrality. This pioneering work established a framework for simulation of ionization effect in rarefied flows. Carlson \& Hassan [36] proposed a scheme to compute the electric field intensity, however, this scheme would suffer significant statistical fluctuation due to the scattering of charged particles. Both approaches have only been applied for one-dimensional stagnation problems because of the computational costs, thus it was difficult and unsuccessful for the DSMC results to compare with flight experiment data [35,37]. The surface geometry of aircraft will strongly influence flow structures including shock standoff distance etc. The flow along the stagnation line can be significantly different for different spacecraft configurations even if all other parameters were the same. Therefore, the real spacecraft geometries should be taken into account for accurate calculations, and it is essential to be able to simulate rarefied air ionization around 3D spacecraft with complex geometries.

Recently, multidimensional DSMC simulations of rarefied ionization have been under rapid development. Ozawa [38] used DSMC and conventional computational fluid dynamics (CFD) methods to simulate rarefied ionization process on Stardust using a simplified axisymmetric configuration. Also using axisymmetric configuration, Boyd 
[39] applied DSMC to study the rarefied ionization of RAMC-II and introduced a special numerical scheme to trace species and electron-ion enforced association. Fan [40] proposed a trace species separation (TSS) scheme to compute the electron density distribution around the RAMC-II which was also simplified to an axisymmetric configuration. Unlike the probabilistic models of traditional DSMC, TTS calculates the particle number densities of trace species from those of common species based on reaction rates. Shevyrin [41] compared the differences among the TCE [35], KSS [42] and QK [43] models for rarefied ionization, where the electron is not modeled as particle and its density is computed as the sum of those ions.

It is still a tough challenge to effectively capture the rarefied ionization effect for $3 \mathrm{D}$ hypervelocity reentry flow, as it needs to overcome two difficulties in order to enable realistic complex 3D simulations. First, restricted by computational resources, the number of simulated molecules in each cell is very limited, often less than the order of ten, thus each cell may not even have one single electron. The presence of an extremely small number of rare species can cause significant numerical fluctuations, which will affect both the determination of the number of molecular collision pair and the electron density sampling. Second, for the simulation of the 3D plasma effect, the electron-ion enforced association would lead to additional computing load and restrict the electron movement, while Carlson and Hassan's method [36] suffers great statistical fluctuation. Meanwhile, if electron is simulated as another species with realistic electron mass, restriction on the time step would be unacceptable. However, Boyd [44] proposed to amplify the mass of electron by three orders of magnitude, i.e. the electrons have almost the same order of speeds with other species after collisions, thus the restriction on the time step can be removed.

In this work, we extend our DSMC method to simulate rarefied atmosphere ionization around 3D complex spacecraft $[5,33,45]$, in which the above artificial amplification in electron mass is adopted, and the mass of ions is adjusted to guarantee mass conservation. A weighting factor scheme for rare species is proposed for the rarefied ionization process, which will mitigate fluctuations on both the determination of the number of molecular collision pair and the electron density sampling. The chemical reactions involving ionization for 11 species air are treated to ensure reproduction of the rare species in resultants and preservation/deletion of common species in reactants according to the weighting factors. The second part of this paper is about the application of the newly developed DSMC modeling method in the hypervelocity reentry flows. The resulting DSMC code is applied to study reentry ionization of spacecraft considering their 3D complex shapes including the RAM-C II and the Stardust, and the effectiveness of the weighting factor scheme is investigated. The rarefied atmosphere ionization during the Chinese lunar exploration capsule reentry is simulated and analyzed with the actual flight test. The remaining parts of this paper are organized as follows: The DSMC algorithm for ionization reaction is introduced in Section 2. The weighting factor scheme for trace species in modelling ionization reaction is proposed in Section 3. The algorithm validation and analysis are presented in Section 4. The numerical simulation and analysis for the rarefied ionization around the Chinese lunar capsule is in Section 5, followed by conclusions and the expectation of the future work in Section 6. 


\section{DSMC modeling for rarefied ionization reaction}

The DSMC method simulates the physics of the Boltzmann equation by following the motions and collisions of a large number of simulated molecules, and their movement and collisions are decoupled over a time step that is smaller than the local mean free time $[5,33,45,46]$. The spatial position coordinates, velocity components and internal energies of the simulated molecules are stored in the computer during computation; and each simulated molecule is assigned a sequence number of local cell index. Their values alter with time due to the movement of simulated molecules and the interactions with boundaries as well as other molecules. When a collision occurs, postcollision velocities are calculated using conservation of momentum and energy. The chemical reaction is implemented as a process of binary molecular collision, usually written as

$$
A+B \rightarrow C+D+E,
$$

where $\mathrm{A}$ and $\mathrm{B}$ are denoted to pre-collision molecules, while $\mathrm{C}$ and $\mathrm{D}$ are the postcollision ones. The chemical reactions with ionization involve dissociation, exchange, associative ionization and its inverse, direct ionization and charge exchange. $E$ represents atom for dissociation or electron for direct ionization. In all other cases, $E$ is null.

The reaction rate coefficients are usually expressed in the Arrhenius form

$$
k(T)=\Lambda T^{\eta} \exp \left(-E_{a} / \kappa T\right),
$$

where $\Lambda$ and $\eta$ are constants, $E_{a}$ is the reaction activation energy, $\kappa$ is the Boltzmann constant and $T$ is temperature. For $E_{c}>E_{a}$, the above $\Lambda$ and $\eta$ relate to reaction probability $P$ by

$$
P=\frac{\pi^{1 / 2} \varepsilon \Lambda T_{r e f}^{\eta}}{2 \sigma_{T, r e f}\left(\kappa T_{r e f}\right)} \frac{\Gamma\left(\bar{\xi}+5 / 2-\omega_{A B}\right)}{\Gamma(\bar{\xi}+\eta+3 / 2)} \times\left(\frac{m_{r}}{2 \kappa T_{r e f}}\right)^{1 / 2} \frac{\left(E_{c}-E_{a}\right)^{\eta+\bar{\xi}+1 / 2}}{E_{c}^{\eta+\bar{\xi}+\omega_{A B}}} .
$$

Here, $\sigma_{T}$ is the total collision cross section, $\varepsilon$ is the symmetric factor whose value is 2 when A equals to B, or 1 otherwise. $T_{r e f}$ is the reference temperature and $\Gamma$ is the Gama function. $\bar{\xi}$ is the average internal degrees of freedom, $\omega_{A B}$ is the average viscosity index, and $m_{r}$ is the reduced mass.

Due to the significant mass difference between electron and usual species, the magnitude of electron speed would be 4 to 5 orders higher than that of other species. If a unified time step is chosen for the motion of electron and molecules, the time step would be too small, resulting in unaffordable computational cost. And if different time steps are used for electron and other species, respectively, although the computational costs could be reduced, complex additional modifications are required for DSMC simulations. An efficient empirical solution is the artificial mass amplification of electron as proposed by Boyd [44]. The principle is to reduce electron speed to nearly the same as other species while the speed of other species will not be affected significantly. If the amplification is too small, the electron speed would still be too much higher than other species. On the contrary, large changes to ion speeds will occur if the amplification is too large as the summation of electron and ion mass should be equal to the original atom or molecule. To striking an appropriate balance, an amplification of electron mass by 3 orders of magnitude is chosen here. Therefore, the electron virtual mass is about 
$3-4 \%$ of atoms, and reduction of ion mass is also up to $3-4 \%$. Moreover, to keep the reaction probability in Eq. (3) and to match the changes of electron mass, the chemical reaction rates involving electrons should be reduced by $\sqrt{1000}$, as shown in Table 1 . For other chemical reactions, the Gupta model $[47,48]$ is adopted.

\section{Weighting factor scheme for trace species in modeling ionization reaction}

Since each cell may not have one single electron, significant fluctuations on both the number of molecular collision pair and the electron density sampling need to be properly dealt with. Instead of using various complex tracking approaches for trace species $[34,49,50]$, a new weighting factor scheme is developed here to model ionization reaction. Different weighting factors are used for different species: the weighting factor is 1 for $\mathrm{N}_{2}, \mathrm{O}_{2}, \mathrm{NO}, \mathrm{N}$ and $\mathrm{O}$, while the $W_{s}$, which is no more than 1 , for $\mathrm{N}_{2}^{+}, \mathrm{O}_{2}^{+}, \mathrm{NO}^{+}$, $\mathrm{N}^{+}, \mathrm{O}^{+}$and electron e, respectively. For convenience, a unified $W_{s}$ is adopted for all trace species in this paper. The weighting factor mainly affects the computation of collisions in two ways, namely, the computation of collision numbers as well as mechanism, and the realization of chemical reactions. Therefore, the following modifications will be introduced in the DSMC code for the simulation of ionization reaction.

\subsection{Weighting factor model for particle collision}

Based on the non-time-counter (NTC) technique of the DSMC method [51], the ionized air species are divided into two groups: group $P$ for electrons only, and group $Q$ for all other species [52]. Neglecting the inter-particle collisions in group $P$, we only need to deal with the inter-particle collisions in group $Q$, and the collisions between group $P$ and $Q$. Without weighting factor, the number of inter-particle collisions in group $Q$ is

$$
\frac{1}{2} N_{Q} \bar{N}_{Q} F_{N}\left(\sigma_{T} c_{r}\right)_{Q Q, \max } \Delta t / V_{c},
$$

where $N_{Q}$ is particle number in group Q. The number of collisions between the particles in groups $P$ and $Q$ is

$$
\frac{1}{2} N_{P} \bar{N}_{Q} F_{N}\left(\sigma_{T} c_{r}\right)_{P Q, \max } \Delta t / V_{c}
$$

Where $N_{P}$ is particle number in group $\mathrm{P}, F_{N}$ denotes the real particle number that a simulating particle represents, $V_{c}$ is the cell volume, and $\left(\sigma_{T} c_{r}\right)_{\mathrm{QQ} \text {, max }}$ and $\left(\sigma_{T} c_{r}\right)_{P Q, \max }$

Table 1 Reaction constants involving electron

\begin{tabular}{lllll}
\hline No & Reaction & $\Lambda$ & $\eta$ & $E_{\text {rea }}$ \\
\hline 1 & $\mathrm{O}+\mathrm{N} \rightarrow \mathrm{NO}^{+}+\mathrm{e}$ & $8.06 \times 10^{-22}$ & 0.37 & $4.422 \times 10^{-19}$ \\
2 & $\mathrm{O}+\mathrm{O} \rightarrow \mathrm{O}_{2}^{+}+\mathrm{e}$ & $2.03 \times 10^{-23}$ & 0.49 & $1.120 \times 10^{-19}$ \\
3 & $\mathrm{~N}+\mathrm{N} \rightarrow \mathrm{N}_{2}^{+}+\mathrm{e}$ & $9.42 \times 10^{-22}$ & 0.77 & $9.340 \times 10^{-19}$ \\
4 & $\mathrm{O}+\mathrm{e} \rightarrow \mathrm{O}^{+}+\mathrm{e}+\mathrm{e}$ & $9.49 \times 10^{-14}$ & 0 & $2.180 \times 10^{-18}$ \\
5 & $\mathrm{~N}+\mathrm{e} \rightarrow \mathrm{N}^{+}+\mathrm{e}+\mathrm{e}$ & $3.16 \times 10^{-16}$ & 0 & $2.330 \times 10^{-18}$ \\
6 & $\mathrm{O}_{2}^{+}+\mathrm{e} \rightarrow \mathrm{O}+\mathrm{O}$ & $1.21 \times 10^{-10}$ & -1.51 & 0 \\
7 & $\mathrm{~N}_{2}^{+}+\mathrm{e} \rightarrow \mathrm{N}+\mathrm{N}$ & $2.08 \times 10^{-11}$ & -1.23 & 0 \\
8 & $\mathrm{NO}^{+}+\mathrm{e} \rightarrow \mathrm{O}+\mathrm{N}$ & $1.27 \times 10^{-10}$ & 1.63 & 0 \\
\hline
\end{tabular}


are the maximum value of the product of cross section and relative speed updated at each time step.

The introduction of weighting factors will increase $N_{P}$ and $N_{Q}$ as well as $\bar{N}_{Q}$ in Eq. (4) and Eq. (5). Traditionally, an array $C S(2, i, j)$ is used to record the particle numbers of species $j$ in cell $i$. Due to the weighting factor, the $N_{Q}$ in Eq. (4) should now be modified as

$$
N_{Q}^{\prime}=\sum_{j} C S(2, i, j) \times W_{j},
$$

where $W_{j}$ is the weighting factor for species $j$, and $N_{P}$ in Eq. (5) becomes

$$
N_{P}^{\prime}=C S(2, i, e) \times W_{e},
$$

where $W_{e}$ is the weighting factor for electron.

As binary collisions are considered in DSMC simulations, the post collision velocities of two particles with identical weight are

$$
\begin{aligned}
& \vec{U}_{1}^{\prime}=\vec{U}_{m}+\frac{m_{2}}{m_{1}+m_{2}} \vec{g}, \\
& \vec{U}_{2}^{\prime}=\vec{U}_{m}-\frac{m_{1}}{m_{1}+m_{2}} \vec{g},
\end{aligned}
$$

where $\vec{U}_{m}$ is the velocity of center of mass of the collision pair, and $\vec{g}$ is their relative velocity. If the two collisional particles have different weighting factors, the velocity of the particle with smaller weight is determined by Eq. (8a) or Eq. (8b). However, the velocity of the particle with the larger weight factor calculated by Eq. (8a) or Eq. (8b) needs to be modified with probability

$$
P=\left\{\begin{array}{cc}
W_{1} / W_{2}, & \text { if } W_{1}<W_{2}, \\
W_{2} / W_{1}, & \text { else },
\end{array}\right.
$$

which means the velocity of the particle still keeps unchanged by the probability of $1-P$.

The above approach cannot exactly conserve momentum and energy in every collision step. However, violence of conservation laws is negligible even after a sufficiently large number of collisions.

\subsection{Weighting factor scheme for chemical reactions}

For the 11-species air model, the chemical reactions process involving rare species can be classified into four types: the reaction between the common species such as associative ionization $\mathrm{N}+\mathrm{O} \rightarrow \mathrm{NO}^{+}+\mathrm{e}$ which produces the rare species; the reaction between the common and rare species such as direct ionization $\mathrm{N}+\mathrm{e} \rightarrow \mathrm{N}^{+}+\mathrm{e}+\mathrm{e}$ which produces the rare species; the reaction between the rare species such as association ionization inverse $\mathrm{NO}^{+}+\mathrm{e} \rightarrow \mathrm{N}+\mathrm{O}$ which produces the common species; and the reaction between the rare and the common species such as $\mathrm{N}_{2}+\mathrm{N}^{+} \rightarrow \mathrm{N}+\mathrm{N}_{2}^{+}$which exchanges charge and produces new species. These four types of reactions are treated separately.

For $\mathrm{N}+\mathrm{O} \rightarrow \mathrm{NO}^{+}+\mathrm{e}$, it reproduces the products of $\mathrm{NO}^{+}$and e by $1 / W_{s}$ copies. And for the inverse association reaction $\mathrm{NO}^{+}+\mathrm{e} \rightarrow \mathrm{N}+\mathrm{O}$, the products are reserved by a probability of $W_{s}$. For $\mathrm{N}+\mathrm{e} \rightarrow \mathrm{N}^{+}+\mathrm{e}+\mathrm{e}$, whose reactant is $\mathrm{N}$ and the product $\mathrm{e}$ is also 
copied by a factor of $1 / W_{s}$ times. However, for $\mathrm{N}_{2}+\mathrm{N}^{+} \rightarrow \mathrm{N}+\mathrm{N}_{2}^{+}$, in which usual and trace species are involved in reactants and products, $\mathrm{N}_{2}$ in the reactants is eliminated and $\mathrm{N}$ in the products is reserved by a probability of $W_{s}$.

The purpose of trace species weighting factor is to increase the number of simulated particles and reduce the statistical fluctuation for the species participated in ionization reaction. Obviously, according to the above weighting factor scheme, the numbers of simulated particles of those species are amplified by a factor of $1 / W_{s}$.

\section{Algorithm validation and discussion}

\subsection{Computational test of DSMC modeling on controlling statistical fluctuation}

In order to validate the present DSMC modeling rules for simulating weakly inhomogeneous flow in simple gases, the planar Couette shear flow from two parallel infinite plates with relative motion placed at a distance of $H$ is simulated and compared with the results of the gas-kinetic unified algorithm (GKUA) [23-27] where we directly solve the Boltzmann-type velocity distribution function equation. The Couette flow is a simple and benchmark boundary-value flow problem. They have the same surface temperature $T_{W}$ and move at a speed $U_{W}$ in opposite directions, as shown in Fig. 1. In this simulation, we choose Ar with temperature $T_{r e f}=273 K, T_{W}=T_{r e f}$ and pressure $P=$ $0.01 \mathrm{~atm}$. The variable soft sphere (VSS) model [32] with $\omega=0.81$ and $\alpha=1.4$ is applied to describe the interaction between molecules. For this Couette flow, we will investigate the change of the statistical noise of the flow velocity with the plate velocity $U_{W}$, the total number of simulated particles $N_{t}$ and the number of sampling $N$. In order to verify the accuracy of the DSMC results, Fig. 2 gives a comparison of the flow velocity distributions between the present DSMC simulations and the Unified Algorithm (GKUA) $[23,53]$ for direct solution of Boltzmann model equation, where the dimensionless flow velocity distributions $U / U_{W}$ along the distance $y / H$ in the upper half of this Couette flow are presented. The DSMC results in Fig. 2 for the Knudsen number of $K n=0.1128$ are respectively corresponding to (a) $U_{W}=0.2 \mathrm{Ma}$ (with $N=2 \times 10^{4}$ and $N_{t}=1000$ ), (b) $N_{t}=64000$ (with $U_{W}=0.025 \mathrm{Ma}$ and $N=2 \times 10^{4}$ ), and (c) $N=128 \times 10^{4}$ (with $U_{W}=$ $0.025 \mathrm{Ma}$ and $N_{t}=1000$ ). It can be shown that since the inflow speed is generally known, in order to reduce the influence of the statistical noise on flow velocity, the

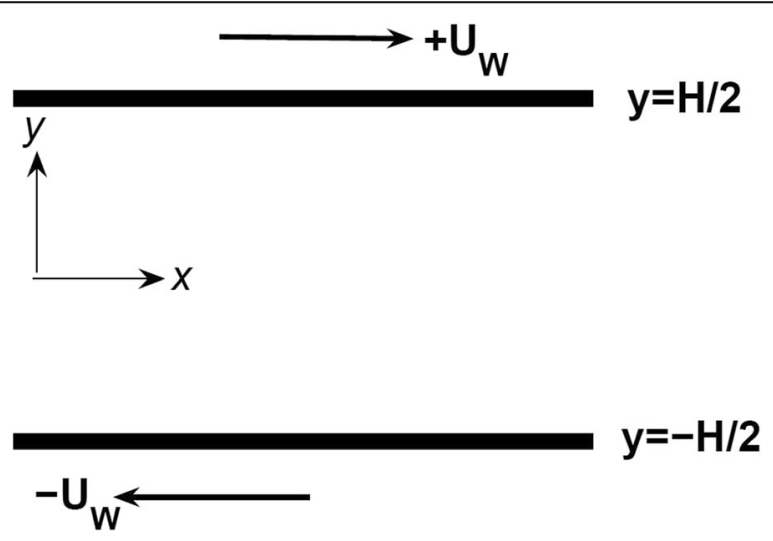

Fig. 1 Computational schematic diagram of the Couette flow 


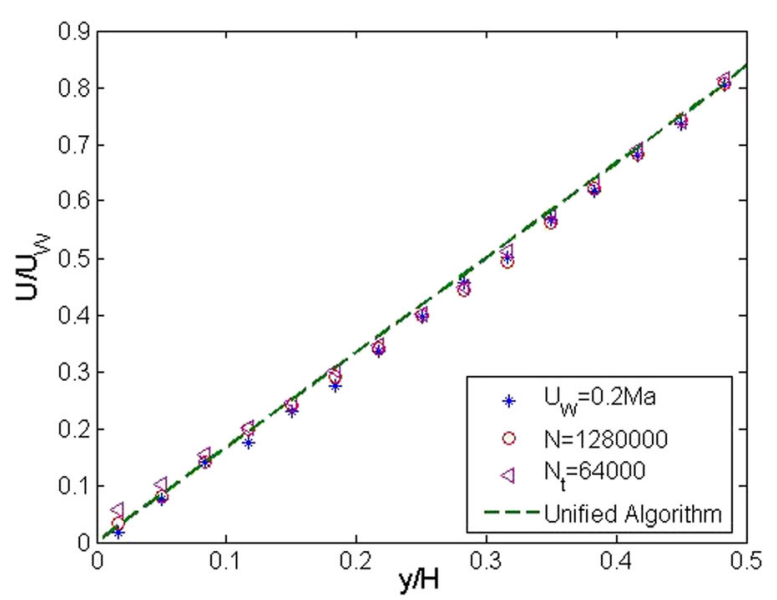

Fig. 2 DSMC results of flow velocity distribution under different conditions with comparison of GKUA

magnitude of $N_{t}$ and $N$ should be chosen as large as possible, and the present DSMC results in satisfying such a rule agree well with the computed distributions from the GKUA within the error range.

The planar Couette shear flow in rarefied transition regime with different Knudsen numbers has been simulated, and Fig. 3 gives the flow velocity distribution of the present DSMC and the GKUA as well as numerical analysis of linearized Boltzmann equation [54] related to $K n=0.1128,1.128$ and 11.28 respectively. And the hard sphere (HS) molecular model is used in the DSMC simulation. The plate speed is $0.2 \mathrm{Ma}$, the total number of simulated particles is $N_{t}=1000$ and the number of sampling is $N=$ $12 \times 10^{4}$. It shows that these results from three approaches agree perfectly. This figure also implies that as the Knudsen number increases, the effect of rarefied gas gradually gets stronger, and the slip velocity between the gas and the wall increases. The comparisons from Figs. 2 and 3 confirm the accurate reliability and practicality of the present DSMC modeling in solving the rarefied flows of simple gases which can control

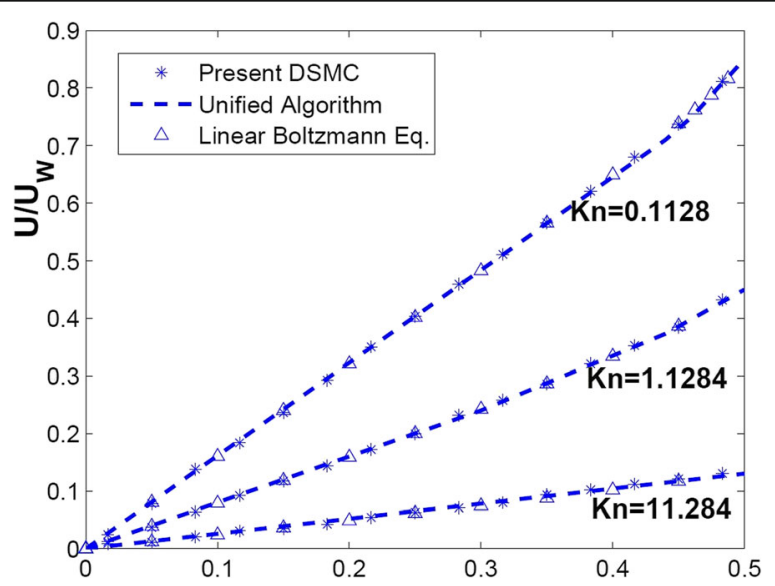

Fig. 3 Flow velocity distributions of the present DSMC under different Knudsen numbers for Couette flow with comparison of the GKUA and the solutions of the linear Boltzmann equation 
Table 2 The test cases for the Shenzhou reentry capsule

\begin{tabular}{lllll}
\hline Case & $M_{\infty}$ & $P_{0}(\mathrm{MPa})$ & $T_{0}(\mathrm{~K})$ & $K n_{\infty}\left(\times 10^{-2}\right)$ \\
\hline 1 & 12.98 & 1.309 & 595 & 1.81 \\
2 & 12.90 & 0.662 & 597 & 2.59 \\
3 & 12.69 & 0.280 & 595 & 3.73 \\
\hline
\end{tabular}

statistical fluctuations to capture the weaker flow information with high resolution, which indicates the validity of the present DSMC algorithm in simulating weakly electron number density distribution around spacecraft during hypervelocity reentry.

\subsection{Shenzhou reentry capsule aerodynamic characteristics of hypersonic reacting gases}

To verify the validity of the present DSMC algorithm with reaction model and rare species weighting scheme for hypersonic non-equilibrium reentry flow, a parallel code has been developed using mesh self-adaption. A series of experiments for the Shenzhou reentry capsule are performed in the low-density wind tunnel as shown in Table 2, to evaluate the present algorithm. Here, $M_{\infty}, P_{0}, T_{0}$ and $K n_{\infty}$ represent the inflow Mach number, the total pressure, the total temperature and the inflow Knudsen number, respectively. The experiment model has a length of $3.75 \mathrm{~cm}$ and a bottom radius of 1.888 $\mathrm{cm}$. The flow media is $\mathrm{N}_{2}$, and the angles of attack (AOA, $\alpha\left(^{\circ}\right)$ ) are set to be $10^{\circ}, 15^{\circ}$, $18^{\circ}, 20^{\circ}, 22^{\circ}, 30^{\circ}$ and $40^{\circ}$ for each case. During the simulation, the fixed wall temperature $300 \mathrm{~K}$ and diffusive reflection boundary condition are adopted.

We focus on the aerodynamic characteristics such as the axial force coefficient $C_{A}$, the lift coefficient $C_{L}$ and the lift-to-drag coefficient $K$, as shown in Fig. 4. The axial force coefficient $C_{A}$ reduces as the AOA increases, with errors of $2.2 \%$ for an AOA of $10^{\circ}$ and $7.9 \%$ for an AOA of $40^{\circ}$. Meanwhile, due to the increase of inflow Knudsen number, the rarefaction effect becomes more significant and leads to the increase of $C_{A}$. The lift coefficient $C_{L}$ has negative values, which does not vary monotonously with AOA but reaches its minimum around an AOA of $30^{\circ}$. The behavior of the lift-to-drag coefficient $K$ is similar as that of $C_{L}$. The excellent agreement between the DSMC calculations and the experimental data demonstrates the reliability of the present algorithm.

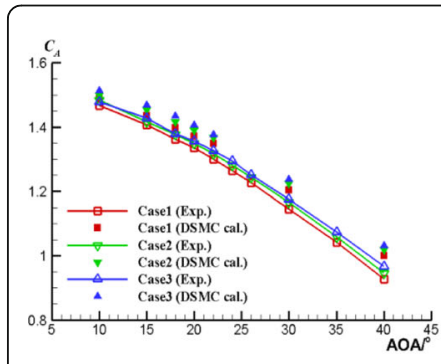

(a) axial force coefficient

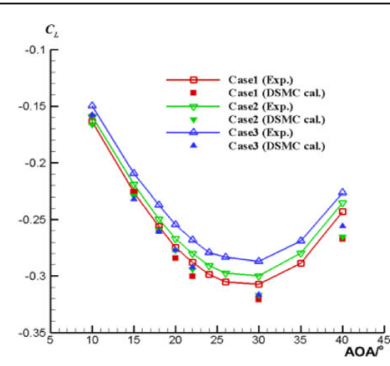

(b) lift coefficient

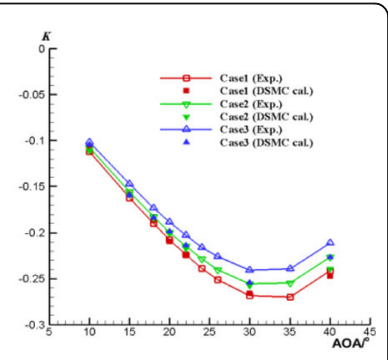

(c) lift-to-drag coefficient

Fig. 4 Aerodynamic characteristics of the Shenzhou reentry capsule. a axial force coefficient. $\mathbf{b}$ lift coefficient. c lift-to-drag coefficient 


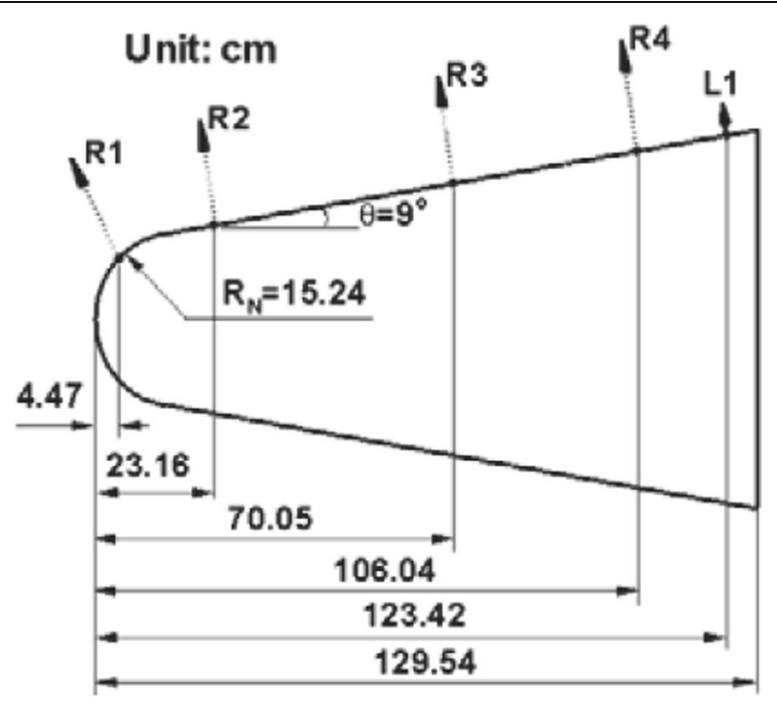

Fig. 5 RAM-C II configuration and probe distribution

\subsection{Ionization reactions for the RAM-C II at $81 \mathrm{~km}$}

The RAM-C (Radio-Wave Attenuation Measurement for Study of Communication Blackout) II flight experiment $[44,55]$ performed in the late 1960 s provides unique experimental data to study ionization in rarefied atmosphere, which is usually used as a classical benchmark case to verify computational models [40-42]. The entry speed of the RAM-C II vehicle is $7.8 \mathrm{~km} / \mathrm{s}$, and its configuration and the probe locations are illustrated in Fig. 5. Here $n_{\infty}, T_{\infty}$ and $V_{\infty}$ represent the inflow molecule number density, the inflow temperature and speed, respectively. The maximum electron density was measured at the four probe locations. The altitude of $81 \mathrm{~km}$ is chosen as a typical test case. The computational setup is summarized in Table 3. The surface temperature is set to be $1000 \mathrm{~K}$ with diffuse reflection of wall boundary condition. Cell self-adaption and sub-cell are used during the simulation, thus the spatial discretization requirement can be satisfied naturally. The flow field around the RAM-C II vehicle is simulated and tested by the present DSMC algorithm with the weighting factor approach of trace species, in which the weighting factor $W_{s}$ is set to be 0.01 . All simulations are performed on a workstation server, with 24 Intel cores and a memory of $192 \mathrm{~Gb}$ on each node.

Figure 6 shows the contour distribution of the flowfield macroscopic properties including number density, temperature, pressure and Mach number, around the RAM-C II vehicle for the flying state at the height of $81 \mathrm{~km}$, where the "Without weighting" and "Weighting scheme" in all of the corresponding captions including the text below denote the modeling of ionization reaction "without" and "with" considering the weighting factor scheme from Section 3 for trace species, respectively. Figure 6 also shows that the weighting factor scheme has negligible effect on the distributions of macroscopic flow parameters. The air is seriously compressed around this vehicle flying at

Table 3 Computational parameters for the case of RAM-C II at $81 \mathrm{~km}$

\begin{tabular}{lllllll}
\hline$n_{\infty}$ & $T_{\infty}$ & $V_{\infty}$ & Domain & Initial cell & Time step & CPU \\
\hline $3.275 \times 10^{20} / \mathrm{m}^{3}$ & $196.7 \mathrm{~K}$ & $7800 \mathrm{~m} / \mathrm{s}$ & {$[-0.25 \mathrm{~m}, 1.35 \mathrm{~m}] \times$} & $360 \times 200 \times 100$ & $1.0 \times 10^{-7} \mathrm{~s}$ & 40 \\
$\left(\mathrm{O}_{2}: 21 \%, \mathrm{~N}_{2}: 79 \%\right)$ & & & {$[-0.5 \mathrm{~m}, 0.5 \mathrm{~m}] \times[0,0.5 \mathrm{~m}]$} & & \\
\hline
\end{tabular}




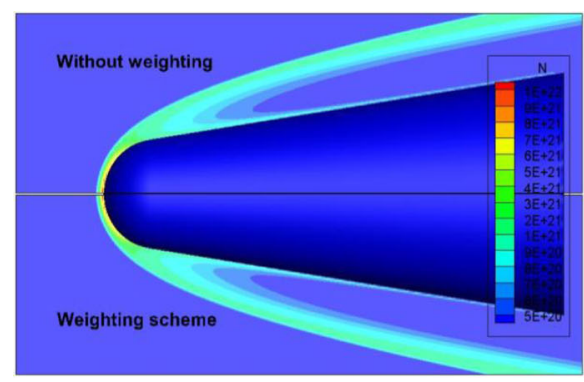

(a) The molecule number density

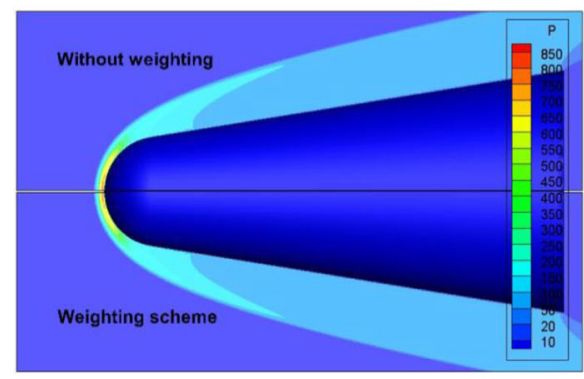

(c) The pressure

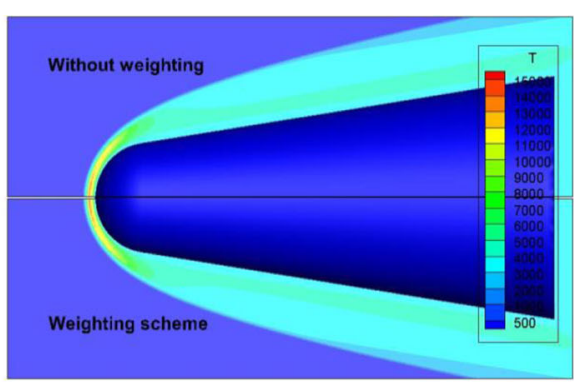

(b) The overall temperature

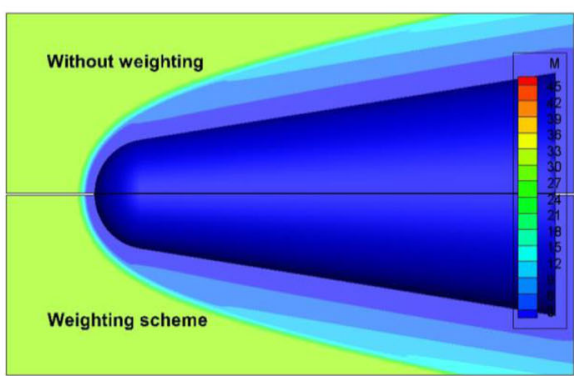

(d) The nominal Mach number

Fig. 6 The macroscopic flow parameters for flow around RAM-C II at $81 \mathrm{~km}$. a The molecule number density. $\mathbf{b}$ The overall temperature. $\mathbf{c}$ The pressure. $\mathbf{d}$ The nominal Mach number

the hypersonic reentry speed. The detached shock is formed near the surface, and the temperature behind the shock reaches as high as $15,000 \mathrm{~K}$. Therefore, chemical reactions, especially ionization, occur in the surface area after the shock wave. Figure 7 presents the contour of electron number density distribution. It is observed that the electron number density concentrates on the surface area behind the shock wave, and the maximum electron number density is about $10^{18} / \mathrm{m}^{3}$, only about $0.01 \%$ of the molecular number density (comparing with Fig. 6a), which means the ionization is extremely weak. Moreover, Fig. 7 demonstrates the effect of the usage of rare species weighting factor scheme on the electron number density distribution, where great fluctuation exists when weighting factor scheme is not used, while the electron number

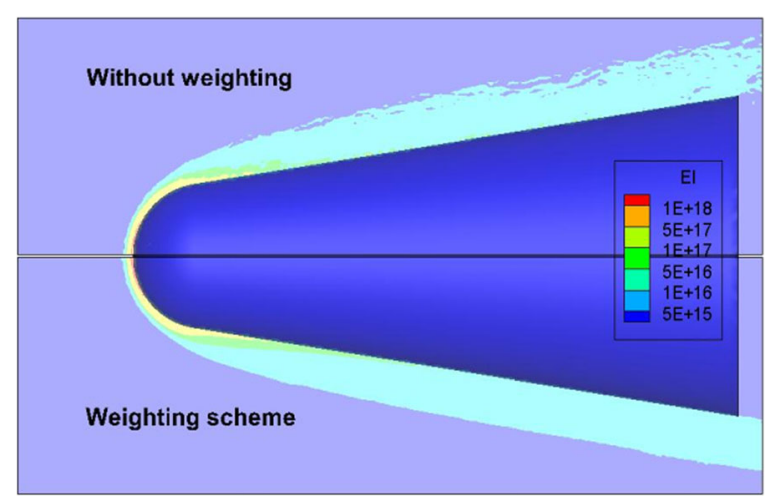

Fig. 7 The electron number density per $\mathrm{m}^{3}$ for RAM-C II at $81 \mathrm{~km}$ 


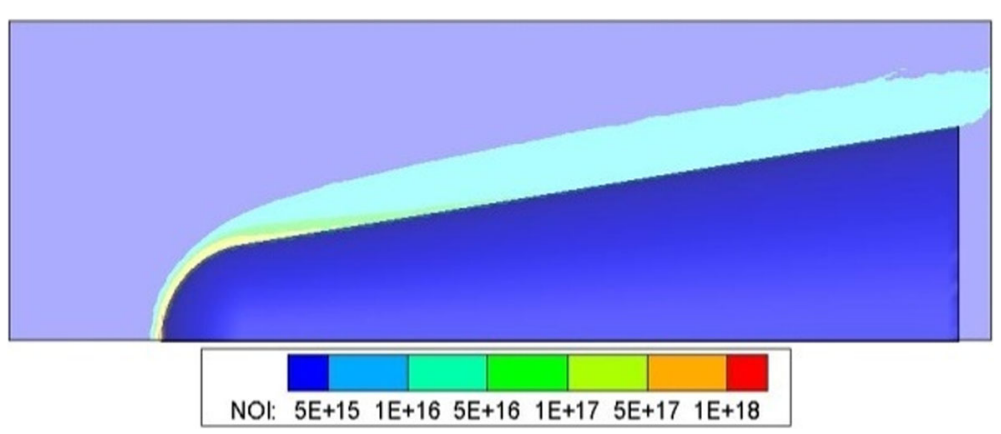

Fig. 8 The $\mathrm{NO}^{+}$number density per $\mathrm{m}^{3}$ for RAM-C II at $81 \mathrm{~km}$

density distribution is smooth enough when the weighting factor scheme is adopted. Interestingly, the electron number density distribution is almost the same as that of $\mathrm{NO}^{+}$, see Fig. 8. This is the reason why the 7-species model [56] is appropriate for moderate hypervelocity reentry, where the main ionization source is associative ionization [44].

The electron number density distributions at the four probe locations as shown in Fig. 9 are obtained by the present DSMC simulations (Symbol " $\diamond$ "), which are also compared with the flight data (Symbol "०") [55] measured by onboard microwave reflectometers. The computed and measured results at all the measurement locations are in excellent agreement at the flying altitude of $81 \mathrm{~km}$, which indicates that the present DSMC algorithm can capture the extremely weak electron number density distribution.

To demonstrate the computing efficiency, we carried out computations for three cases: without chemical reaction, chemical reaction without ionization, and chemical reaction with ionization. For each case, the program runs 20,000 steps to achieve the assumed stable state before sampling, then another 40,000 steps to obtain the routine statistical sampling for the macroscopic flow variables. The CPU time is given in Table 4, which shows that the computational cost of each case differs slightly. Comparing with that in Ref. [40], where a DELL cluster with 64 processors was used and $20 \mathrm{~h}$ of CPU time was consumed, the present proposed model and the developed code can be said to be highly

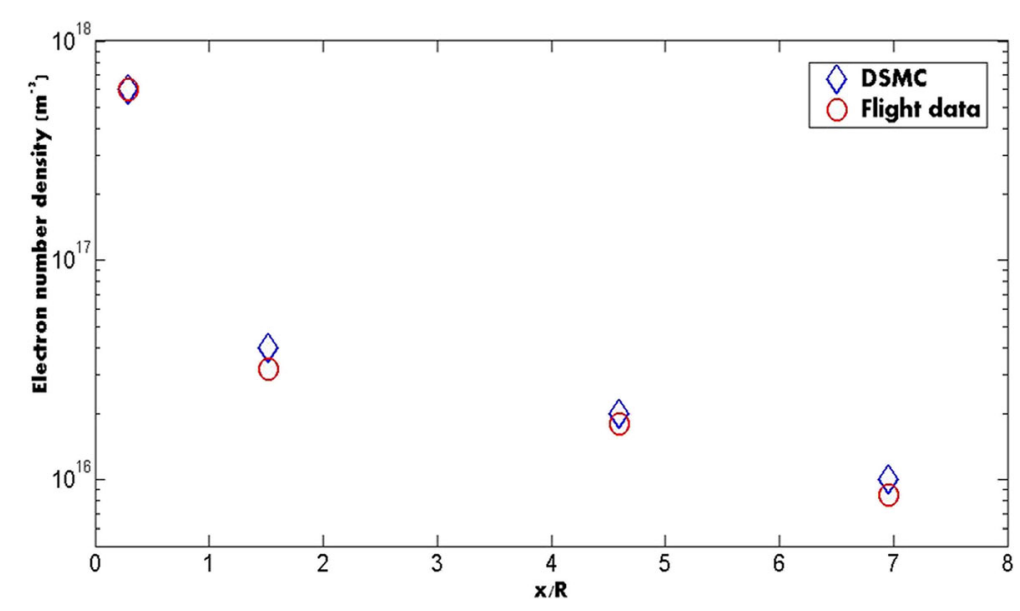

Fig. 9 The maximum electron number density comparison at the probe locations for RAM-C II at $81 \mathrm{~km}$ 
Table 4 CPU time for the test cases

\begin{tabular}{llll}
\hline Case & Without reaction & Without ionization & With ionization \\
\hline CPU time & $21 \mathrm{hr} 44 \mathrm{~min}$ & $22 \mathrm{hr02min}$ & 22hr06min \\
\hline
\end{tabular}

efficient. On the other hand, the case in Ref. [40] is 2D computation, and the present computation is 3D with different CPU time, in which the total number of simulated particles in the present case is about $5 \times 10^{7}$, while that in Ref. [40] is $10^{7}$.

\subsection{Ionization reactions during the Stardust hypervelocity reentry}

Since the Stardust has the highest manmade reentry speed, ionization is more significant. It has a nose radius of $0.22 \mathrm{~m}$ and a head with half cone-angle of $59.5^{\circ}$. The total length is $0.5 \mathrm{~m}$ and the bottom radius is $0.25 \mathrm{~m}$, and the configuration is illustrated in Fig. 10. Similarly, the computational conditions are shown in Table 5. To compare with Ref. [3], we compute the reentry case at $80 \mathrm{~km}$. The computational domain is [- $0.3 \mathrm{~m}$, $0.6 \mathrm{~m}] \times[-0.6 \mathrm{~m}, 0.6 \mathrm{~m}] \times[0,0.6 \mathrm{~m}]$, with 87 million simulated particles initially. The weighting factor $W_{s}$ of trace species is set to be 0.1 . Surface temperature is set to be $1000 \mathrm{~K}$, and the wall is set to be diffusive. Cell self-adaption and sub-cell are also used.

The macroscopic flow variables are shown in Fig. 11, which suggests that the air is more seriously compressed as the free-stream speed is up to $12.8 \mathrm{~km} / \mathrm{s}$. However, the detached shock is formed further away from the Stardust surface than that of the RAM-C II, as the Stardust is a larger blunt vehicle with the head half cone-angle of $59.5^{\circ}$ and a wider area of strong disturbance. The highest temperature behind the shock reaches above $36,000 \mathrm{~K}$, indicating that serious ionization reactions occur near

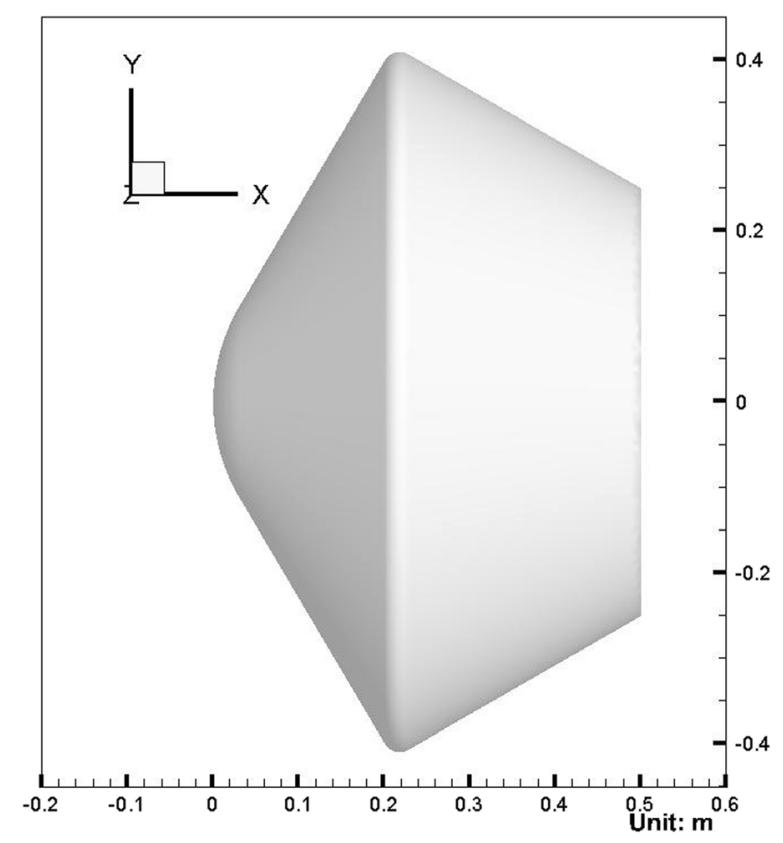

Fig. 10 Configuration of the Stardust 
Table 5 Computational parameters for the case of Stardust at $H=80 \mathrm{~km}$ for $V_{\infty}=12.8 \mathrm{~km} / \mathrm{s}$

\begin{tabular}{lllllll}
\hline$n_{\infty}$ & $T_{\infty}$ & $V_{\infty}$ & Domain & Initial cell & Time step & CPU \\
\hline $3.275 \times 10^{20} / \mathrm{m}^{3}$ & $196.7 \mathrm{~K}$ & $12,800 \mathrm{~m} / \mathrm{s}$ & {$[-0.3 \mathrm{~m}, 0.6 \mathrm{~m}]$} & 270 & $1.0 \times 10^{-7} \mathrm{~s}$ & 90 \\
& & & $\times[-0.6 \mathrm{~m}, 0.6 \mathrm{~m}]$ & $\times 360$ & & \\
$\left(\mathrm{O}_{2}: 21 \%, \mathrm{~N}_{2}: 79 \%\right)$ & & & $\times[0,0.6 \mathrm{~m}]$ & $\times 180$ & & \\
\hline
\end{tabular}

the surface region. The negligible influence of weighting factor scheme on macroscopic flow variables is also confirmed, see Fig. 11.

The major concern is still the electron number density distribution in the flowfield, especially near the front surface of the Stardust, as shown in Fig. 12a. The maximum electron density is larger than $5.0 \times 10^{20} / \mathrm{m}^{3}$, which implies that the ionization can reach $3 \%$ for spacecraft reentering with an extremely high speed of $12.8 \mathrm{~km} / \mathrm{s}$. Figure $12 \mathrm{~b}$ and $\mathrm{c}$ also show the number density distributions of $\mathrm{N}^{+}$and $\mathrm{O}^{+}$. In contrast with the case of the RAM-C II, the main ionization source is the direct ionization of $\mathrm{N}$ and $\mathrm{O}$ atoms. The ionization reaction area is larger, which almost envelops

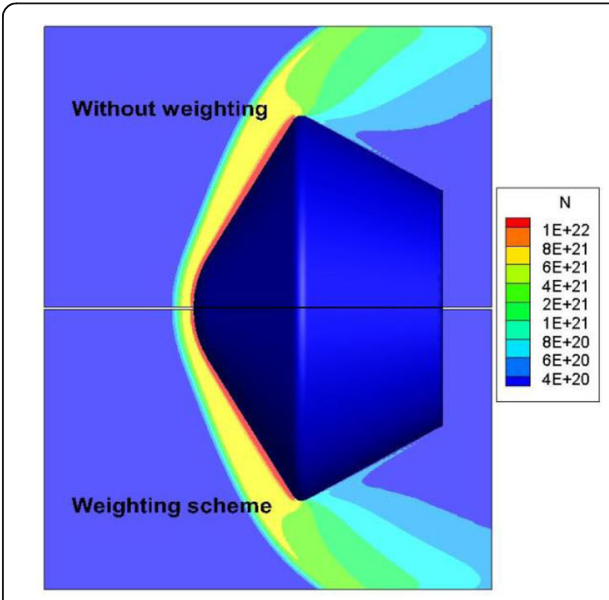

(a) The molecule number density

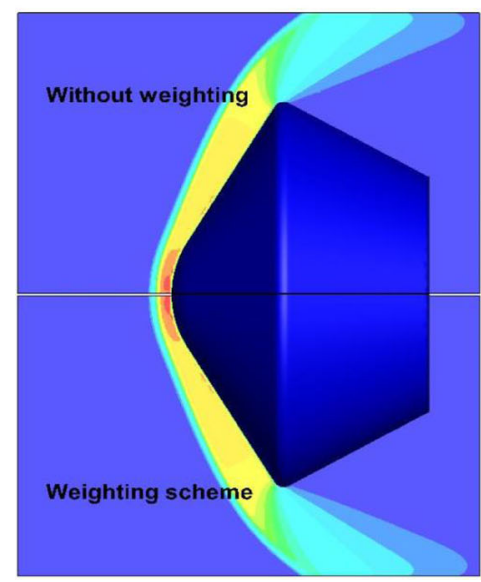

(c) The pressure

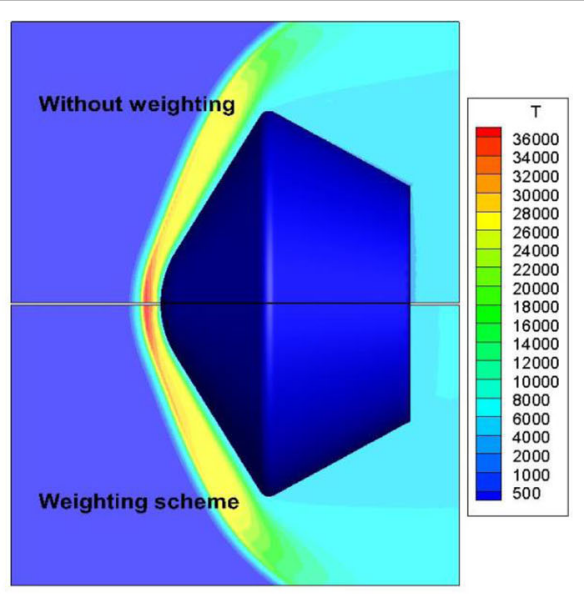

(b) The overall temperature

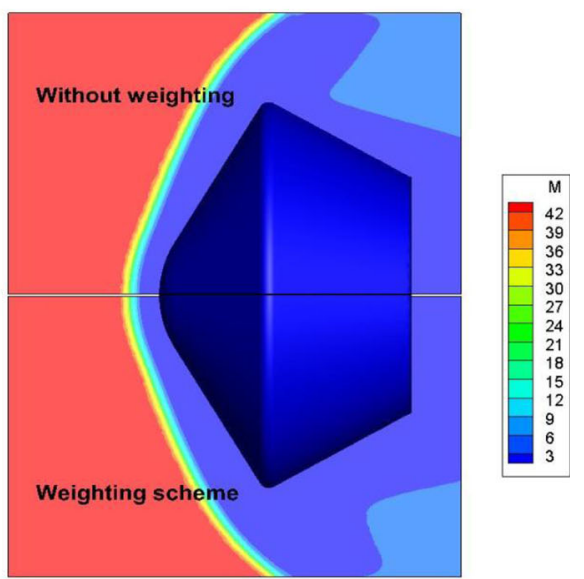

(d) The nominal Mach number

Fig. 11 The distribution of flowfield macroscopic variables around Stardust at $H=80 \mathrm{~km}, V_{\infty}=12.8 \mathrm{~km} / \mathrm{s}$. a The molecule number density. $\mathbf{b}$ The overall temperature. $\mathbf{c}$ The pressure. $\mathbf{d}$ The nominal Mach number 


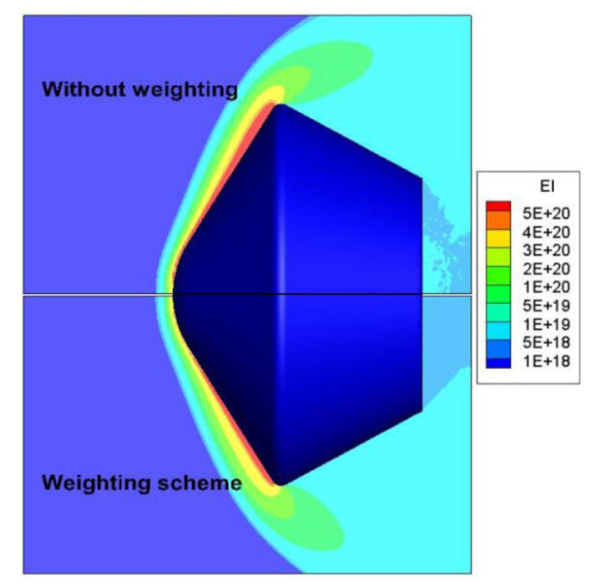

(a) The electron number density

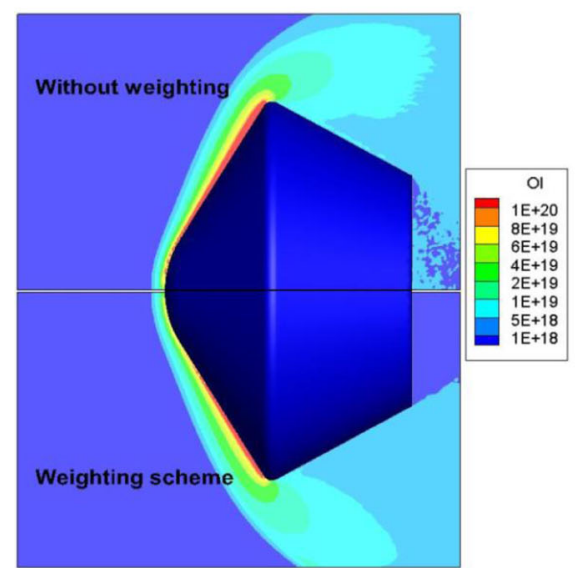

(c) The number density of $\mathrm{O}^{+}$

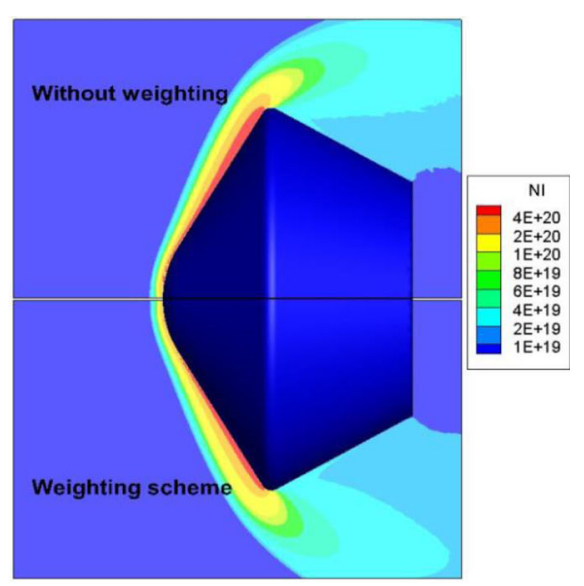

(b) The number density of $\mathrm{N}^{+}$

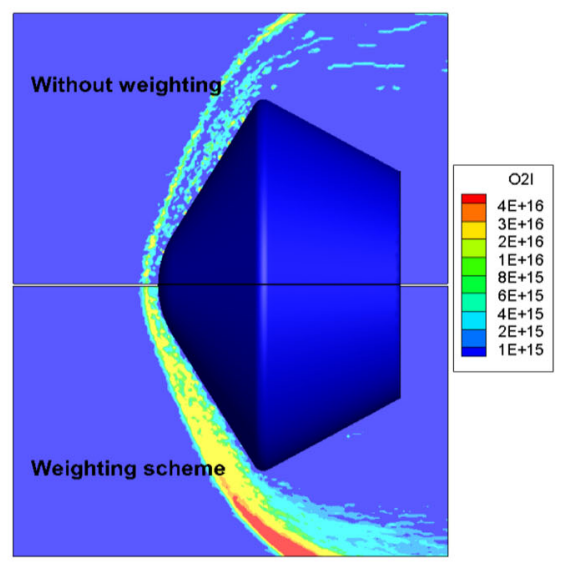

(d) The number density of $\mathrm{O}_{2}^{+}$

Fig. 12 The number densities for electron, $\mathrm{N}^{+}, \mathrm{O}^{+}$and $\mathrm{O}_{2}{ }^{+}$around Stardust at $\mathrm{H}=80 \mathrm{~km}, \mathrm{~V}_{\infty}=12.8 \mathrm{~km} / \mathrm{s}$. a The electron number density. $\mathbf{b}$ The number density of $\mathrm{N}^{+}$. $\mathbf{c}$ The number density of $\mathrm{O}^{+}$. $\mathbf{d}$ The number density of $\mathrm{O}_{2}^{+}$

the head cone surface so that the communication blackout is formed for the Stardust reentry flow at $H=80 \mathrm{~km}$ flying with a speed of $12.8 \mathrm{~km} / \mathrm{s}$. Due to the relatively stronger ionization, the fluctuation of rare species without weighting scheme is smaller. However, the usage of rare species weighting factor scheme can still improve the smoothness of their distribution contours, especially, for the extremely rare species such as $\mathrm{O}_{2}{ }^{+}$.

Figure 13 shows the comparison of electron number density distribution in the flowfield between the present 3D calculation (lower part) and the axis-symmetric simplified model (upper part) [3]. In general, the electron number density contours agree well, although small difference exists in its thickness and maximum value. At this zero angle of attack, both methods predict almost the same standoff distance for the detached bow wave. However, quantitative comparisons of the electron, $\mathrm{N}^{+}$and $\mathrm{O}^{+}$number density profiles along the stagnation line show significant difference, see Fig. 14, especially for the rare components i.e. $\mathrm{N}^{+}$and $\mathrm{O}^{+}$. And Fig. 14 confirms the main ionization 


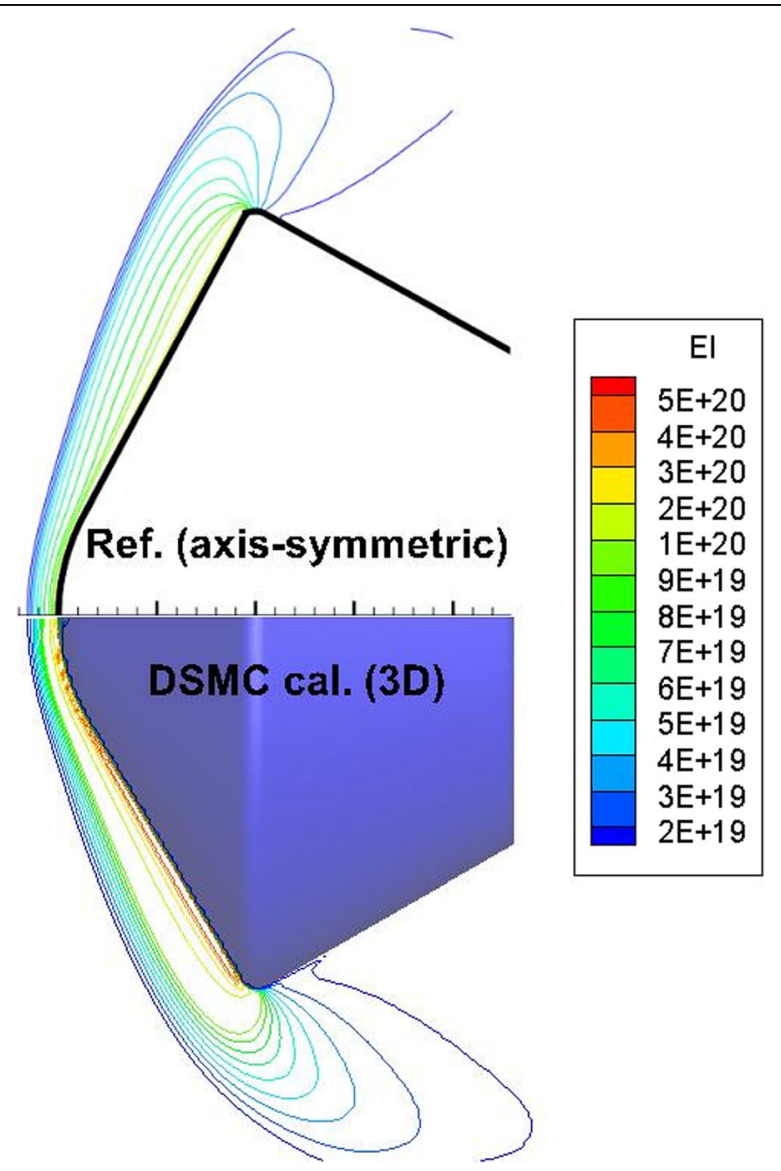

Fig. 13 Comparison of the present 3D calculation and axis-symmetric results [3] for Stardust at $80 \mathrm{~km}$

sources as shown in Fig. 12, different from the prediction of the axis-symmetric model [3], where a significant amount of $\mathrm{NO}^{+}$still exists. From the computation and comparison of the ionization reactions of the RAM-C II and Stardust reentry flows, the present three-dimensional DSMC algorithm can capture fine flow field better than the axissymmetric DSMC model. The weighting factor scheme of trace species is essential to describe weak flow information of the electron, $\mathrm{N}^{+}$and $\mathrm{O}^{+}$number densities.

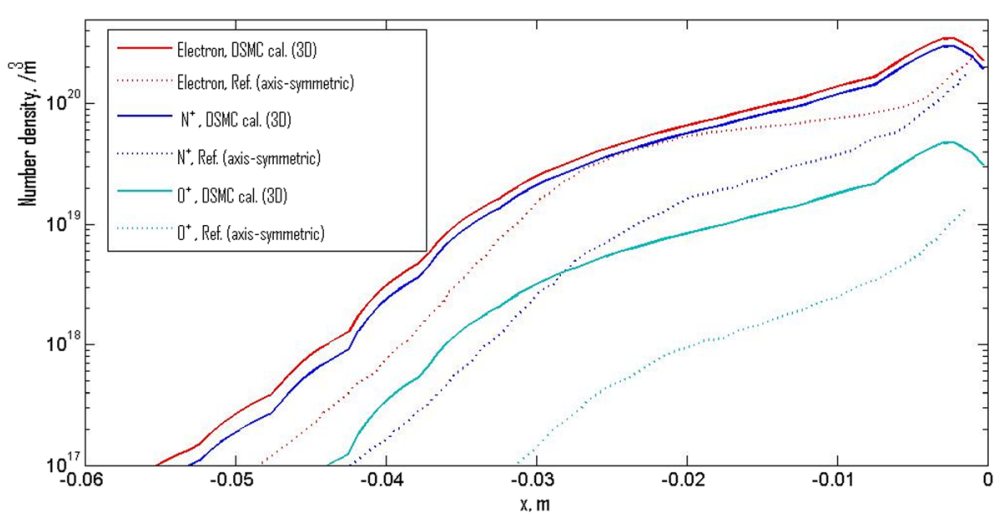

Fig. 14 Quantitative comparison of the electron, $\mathrm{N}^{+}$and $\mathrm{O}^{+}$density distributions along the stagnation line for the Stardust 


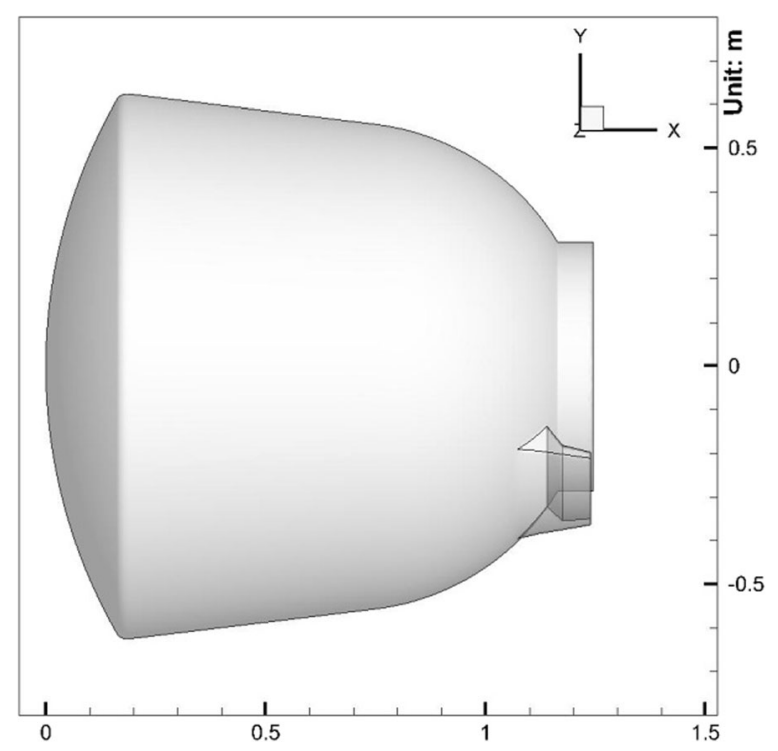

Fig. 15 Vehicle configuration of the Chinese lunar reentry capsule

\section{Rarefied ionization analysis and forecast for the Chinese lunar capsule reentry}

The Chinese lunar capsule returned on November 1, 2014, and its configuration is illustrated in Fig. 15. Since it re-entered the earth atmosphere with double jump reentry from the second cosmic velocity to the first cosmic speed, its atmospheric ionization was significant and suffered a much longer time period of the radio black-out with two access communication interruptions from high rarefied flow to near space flight environment. Due to the existence of its ear piece accessories and the flying angle of attack, flow simulations of a 3D realistic geometry are required. Here, the ionization process is numerically forecasted by the present DSMC modeling with three different stages: (1) the first reentry, between the altitudes of 90 and $97 \mathrm{~km}$, (2) the first jump-out, between 80 and $90 \mathrm{~km}$, (3) the second reentry, between 80 and $85 \mathrm{~km}$. For all the three cases, diffusive wall reflection with fixed temperature $300 \mathrm{~K}$ is used, and cell self-adaption and sub-cell are also adopted.

\subsection{Ionization analysis for the first reentry}

According to its trajectory, typical related parameters are listed in Table 6, with an initial cell system of $240 \times 250 \times 250$, and 60 CPUs carry out the parallel computation

Table 6 Typical related parameters of the lunar exploration type capsule for the first reentry

\begin{tabular}{llll}
\hline Height & $90 \mathrm{~km}$ & $95 \mathrm{~km}$ & $97 \mathrm{~km}$ \\
\hline Inflow temperature & $189.5 \mathrm{~K}$ & $186 \mathrm{~K}$ & $183 \mathrm{~K}$ \\
Inflow number density & $7.116 \times 10^{19} / \mathrm{m}^{3}$ & $2.920 \times 10^{19} / \mathrm{m}^{3}$ & $2.038 \times 10^{19} / \mathrm{m}^{3}$ \\
Inflow Knudsen number & $1.9 \times 10^{-2}$ & $4.6 \times 10^{-2}$ & $6.6 \times 10^{-2}$ \\
Inflow speed & $10,663 \mathrm{~m} / \mathrm{s}$ & $10,663 \mathrm{~m} / \mathrm{s}$ & $10,662 \mathrm{~m} / \mathrm{s}$ \\
Angle of attack(AoA) & $-20^{\circ}$ & $-23^{\circ}$ & $-25^{\circ}$ \\
\hline
\end{tabular}



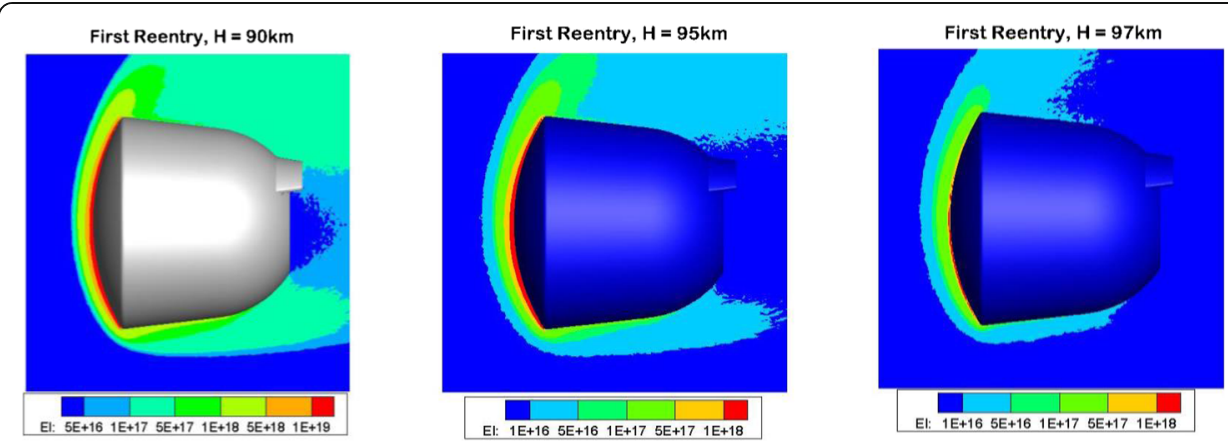

Fig. 16 Electron number density distributions of lunar exploration type capsule at different altitudes for the first reentry (Unit: $\left./ \mathrm{m}^{3}\right)$

along $\mathrm{x}$ direction. The inflow gas is composed of $79 \% \mathrm{~N}_{2}$ and $21 \% \mathrm{O}_{2}$. The time step is $10^{-7} \mathrm{~s}$, and the statistical sampling starts from 40,000 time steps after initialization. The weighting factor for rare species is 0.1 .

This study mainly concerns the electron number density distribution around the capsule. For the three typical heights in Table 6, the corresponding electron number density distributions are shown in Fig. 16, with maximum values $5.216 \times 10^{19} / \mathrm{m}^{3}$, $5.047 \times 10^{18} / \mathrm{m}^{3}$ and $1.988 \times 10^{18} / \mathrm{m}^{3}$. Since the corresponding maximum particle number densities are $9.35 \times 10^{21} / \mathrm{m}^{3}, 3.19 \times 10^{21} / \mathrm{m}^{3}$ and $2.05 \times 10^{21} / \mathrm{m}^{3}$, the ionization degrees are extremely low. For these cases, the ionization degree can be defined as the ratio between electron numbers and particle numbers in the cell, which shows the orders of $1 \%$ to $1 \%$ o for these heights.

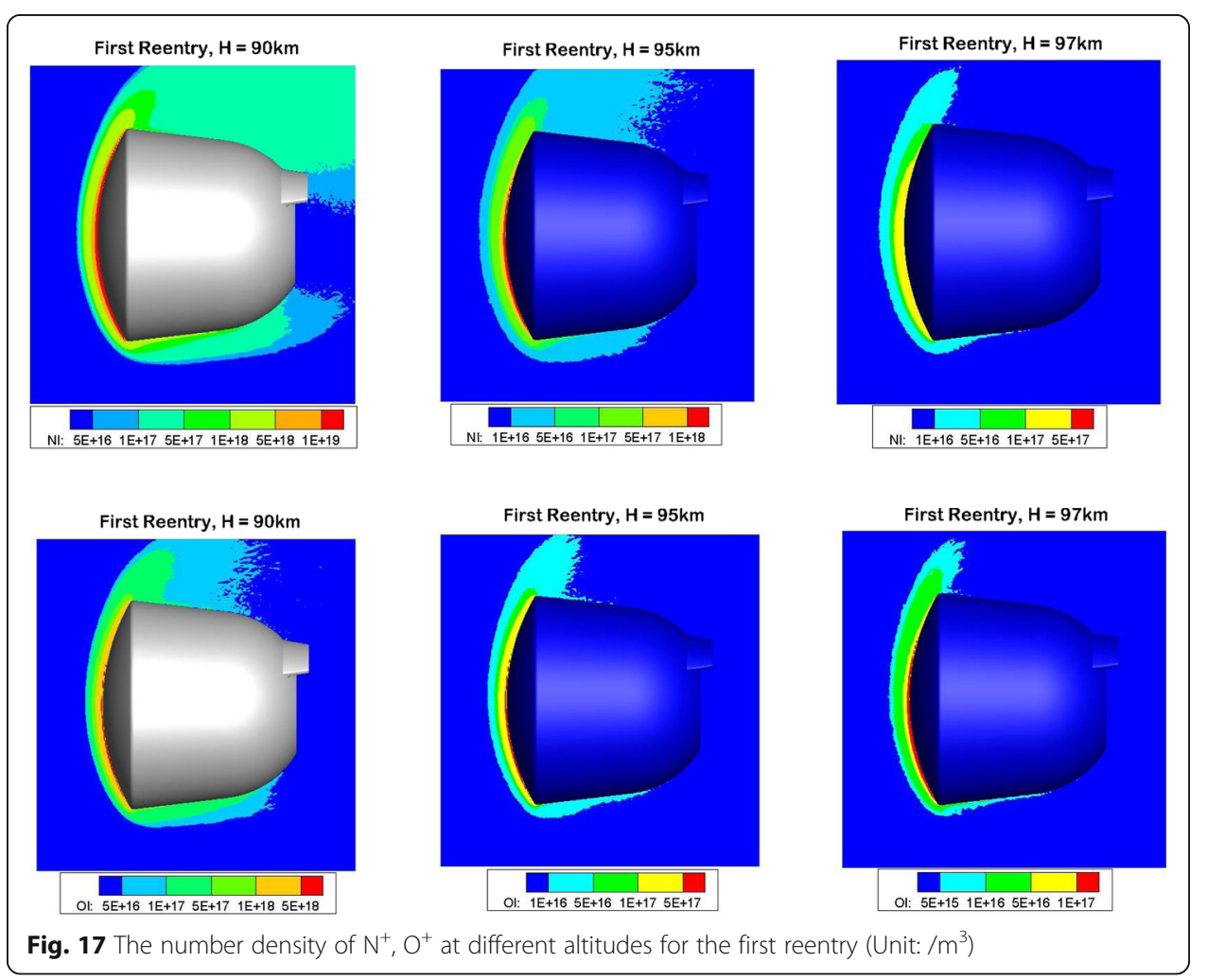



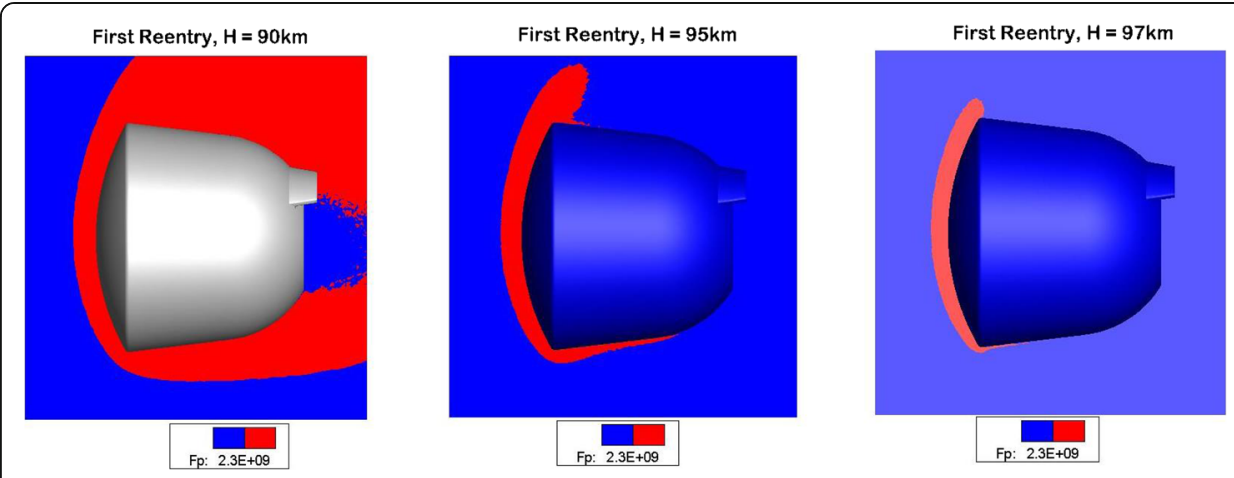

Fig. 18 The corresponding electromagnetic wave frequency of lunar exploration type capsule at different altitudes for the first reentry (Unit: $\mathrm{Hz})$

Similar to the case of the Stardust, the main ionization source is the direct ionization due to the collisions between $\mathrm{N} / \mathrm{O}$ atoms and neural atoms/molecules, and this point can be supported by the number density distributions of $\mathrm{N}^{+}$and $\mathrm{O}^{+}$in Fig. 17. Here, the sum of $\mathrm{N}^{+}$and $\mathrm{O}^{+}$number densities is almost equal to that of the electron, and the contribution of $\mathrm{NO}^{+}$is in the order of $1 \%$, while the contributions of $\mathrm{N}_{2}{ }^{+}$and $\mathrm{O}_{2}{ }^{+}$are lower for at least one order of magnitude.

For the interest of radio communication design, the main focus is the electromagnetic shielding. And for the weak plasma formed by the rarefied atmosphere ionization, the plasma electron oscillation frequency [57] is determined by

$$
\omega_{p e}=\sqrt{\frac{n_{e} e^{2}}{m_{e} \varepsilon_{0}}},
$$

where $n_{e}$ is the electron number density, $e$ is the electron charge, $m_{e}$ is the electron mass, and $\varepsilon_{0}$ is the dielectric constant. If $n_{e}$ is expressed in unit of $\mathrm{cm}^{-3}$, the corresponding radio frequency can be approximated as

$$
f_{p e}=\frac{\omega}{2 \pi} \approx 9000 \sqrt{n_{e}}
$$

Since the capsule returns with an AoA between $-20^{\circ}$ and $-25^{\circ}$, and there is a certain angle between the capsule and the ground radar station, the electron number density contour that envelopes the whole bottom part of the capsule will lead to radio blackout. The ground radar frequency is about $2.3 \mathrm{GHz}$, which is plotted in Fig. 18 and reveals that the complete communication blackout will start at about between 95 and $97 \mathrm{~km}$, and the flight observation value is about $97.1 \mathrm{~km}$.

Table 7 Typical related parameters of the lunar exploration type capsule for the first jump-out

\begin{tabular}{llll}
\hline Height & $80 \mathrm{~km}$ & $85 \mathrm{~km}$ & $90 \mathrm{~km}$ \\
\hline Inflow temperature & $197 \mathrm{~K}$ & $187 \mathrm{~K}$ & $183 \mathrm{~K}$ \\
Inflow number density & $3.838 \times 10^{20} / \mathrm{m}^{3}$ & $1.709 \times 10^{20} / \mathrm{m}^{3}$ & $2.038 \times 10^{19} / \mathrm{m}^{3}$ \\
Inflow Knudsen number & $3.5 \times 10^{-3}$ & $7.8 \times 10^{-3}$ & $1.9 \times 10^{-2}$ \\
Inflow speed & $7292 \mathrm{~m} / \mathrm{s}$ & $7245 \mathrm{~m} / \mathrm{s}$ & $7217 \mathrm{~m} / \mathrm{s}$ \\
Angle of attack(AoA) & $-22^{\circ}$ & $-22^{\circ}$ & $-22.5^{\circ}$ \\
\hline
\end{tabular}




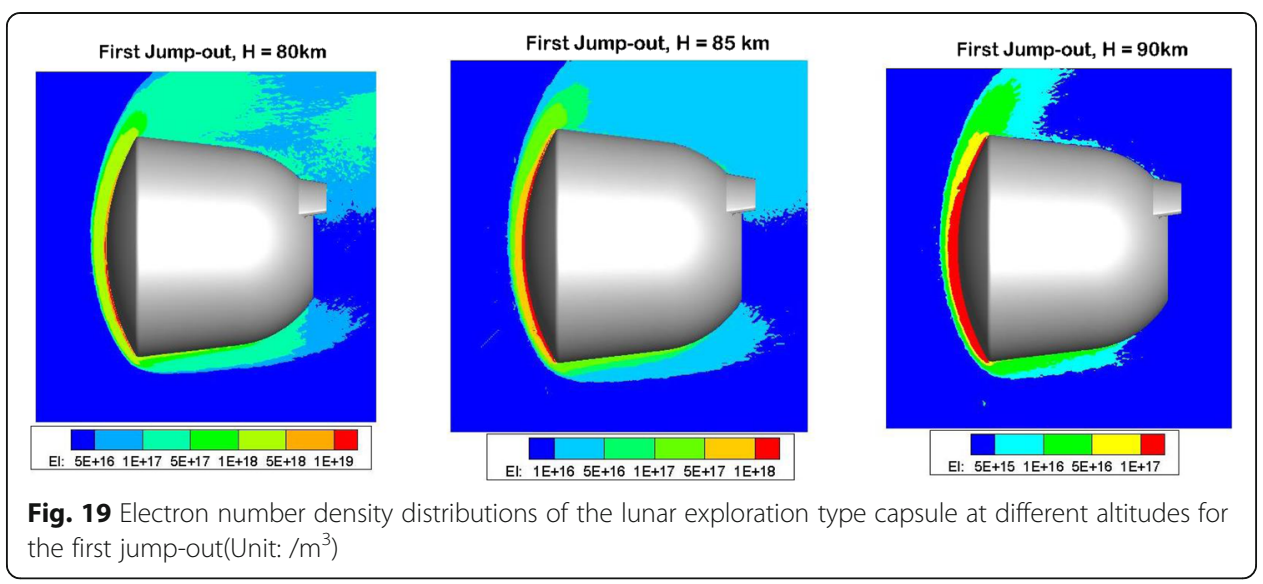

\section{2 lonization analysis for the first jump-out}

Similarly, the typical parameters are shown in Table 7, with an initial cell system of $480 \times 500 \times 500$. Here, the weighting factor for rare species is 0.01 , while other conditions are the same as in Section 5.1.

The electron number densities and electromagnetic wave frequency corresponding to Figs. 16 and 18 are given in Figs. 19 and 20. Since that the reentry speed reduces to lower than the first cosmic speed, the electron number density at $90 \mathrm{~km}$ decreases for about one order of magnitude, and the degree of ionization reduces significantly. Thus, the demand for weighting factor scheme behaves more serious, so that the smaller weighting fact is employed for the rare species in the present computation. According to Fig. 20, the complete communication blackout will occur at the altitude of $85 \mathrm{~km}$, or a little higher.

The main ionization source is still the direct ionization due to the collisions between $\mathrm{N} / \mathrm{O}$ atoms and neural atoms / molecules, here the further discussion will not continue due to the length of the paper.

\subsection{Ionization analysis for the second reentry}

Similarly again, typical parameters are shown in Table 8 . All other parameters are set as same as those in Section 5.2.

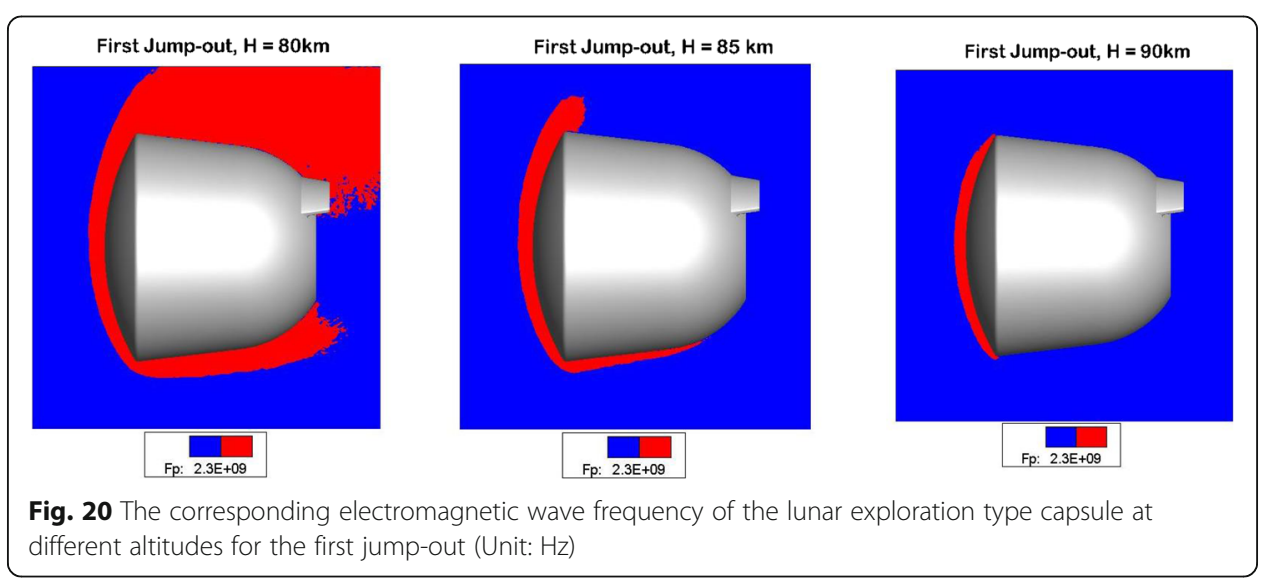


Table 8 Typical related parameters of the lunar exploration type capsule for the second reentry

\begin{tabular}{lll}
\hline Height & $80 \mathrm{~km}$ & $85 \mathrm{~km}$ \\
\hline Inflow temperature & $204 \mathrm{~K}$ & $188 \mathrm{~K}$ \\
Inflow number density & $3.838 \times 10^{20} / \mathrm{m}^{3}$ & $1.709 \times 10^{20} / \mathrm{m}^{3}$ \\
Inflow Knudsen number & $3.5 \times 10^{-3}$ & $7.8 \times 10^{-3}$ \\
Inflow speed & $7161 \mathrm{~m} / \mathrm{s}$ & $7127 \mathrm{~m} / \mathrm{s}$ \\
Angle of attack(AoA) & $-22.5^{\circ}$ & $-24^{\circ}$ \\
\hline
\end{tabular}

The electron number densities and electromagnetic wave frequency are given in Figs. 21 and 22. The electron number density distribution in Fig. 21 differs slightly with that in Fig. 19, and the reason is that the speed difference is not significant at the same altitude, comparing with the first jump-out. Figure 22 shows that, for the S-band electromagnetic wave at the frequency of $2.3 \mathrm{GHz}$, the complete communication blackout will take place at about $85 \mathrm{~km}$, or a little lower. And the conclusion for main ionization source is still the same.

\subsection{Comparison of the present numerical forecast with the flight observation}

In summary, the comparisons of complete communication blackout between the present forecasts and the flight observations can be shown in the following Table 9. Obviously, the differences between the present numerical forecasts and the observed value from the flight test of the Chinese lunar exploration capsule launched in 2014 are on the order of $2 \mathrm{~km}$, which reveals the excellent capabilities of the present numerical forecast of the ionization DSMC modeling based on the rare species weighting factor schemes.

\section{Conclusion}

Rarefied gas ionization effects on aerodynamics of spacecraft hypervelocity reentry are of the greatest practical interest. A DSMC modeling with the weighting factor approach

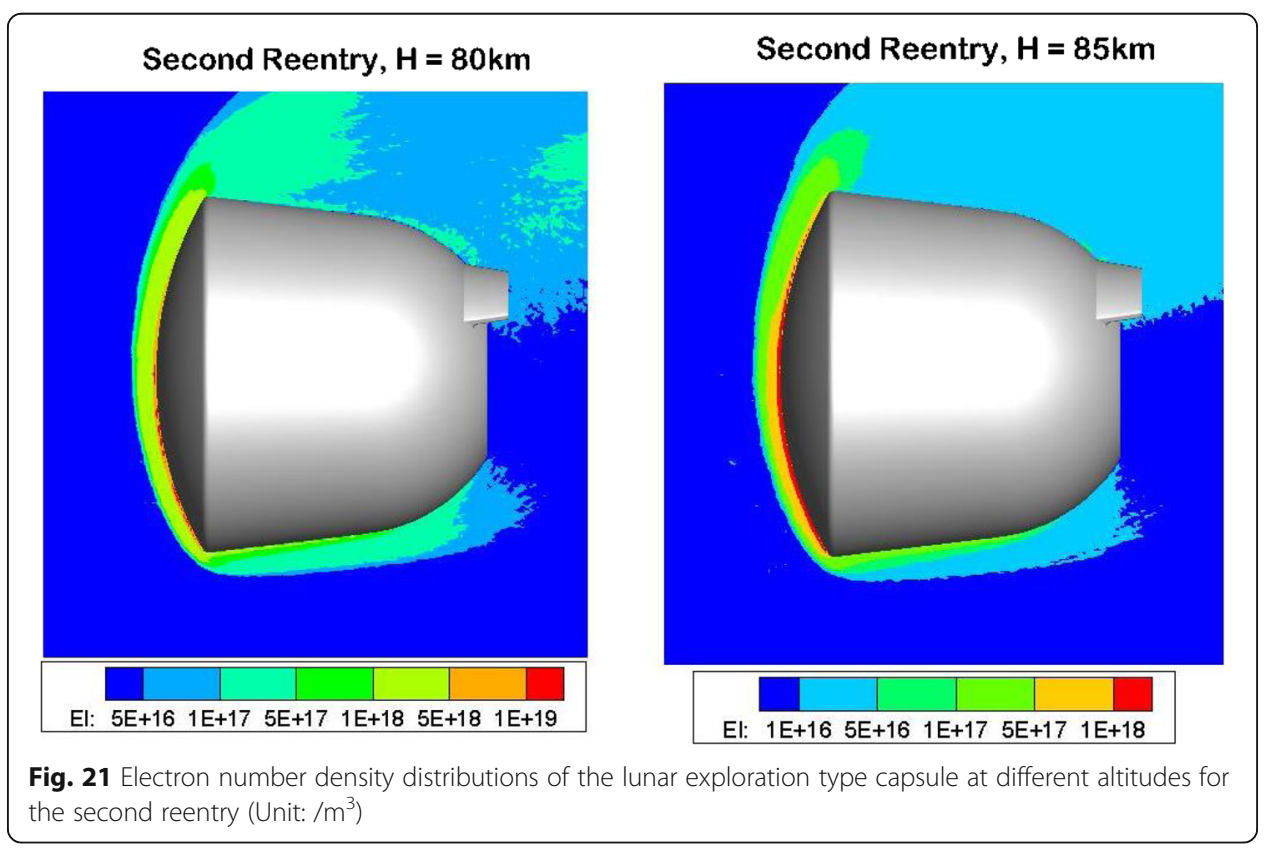




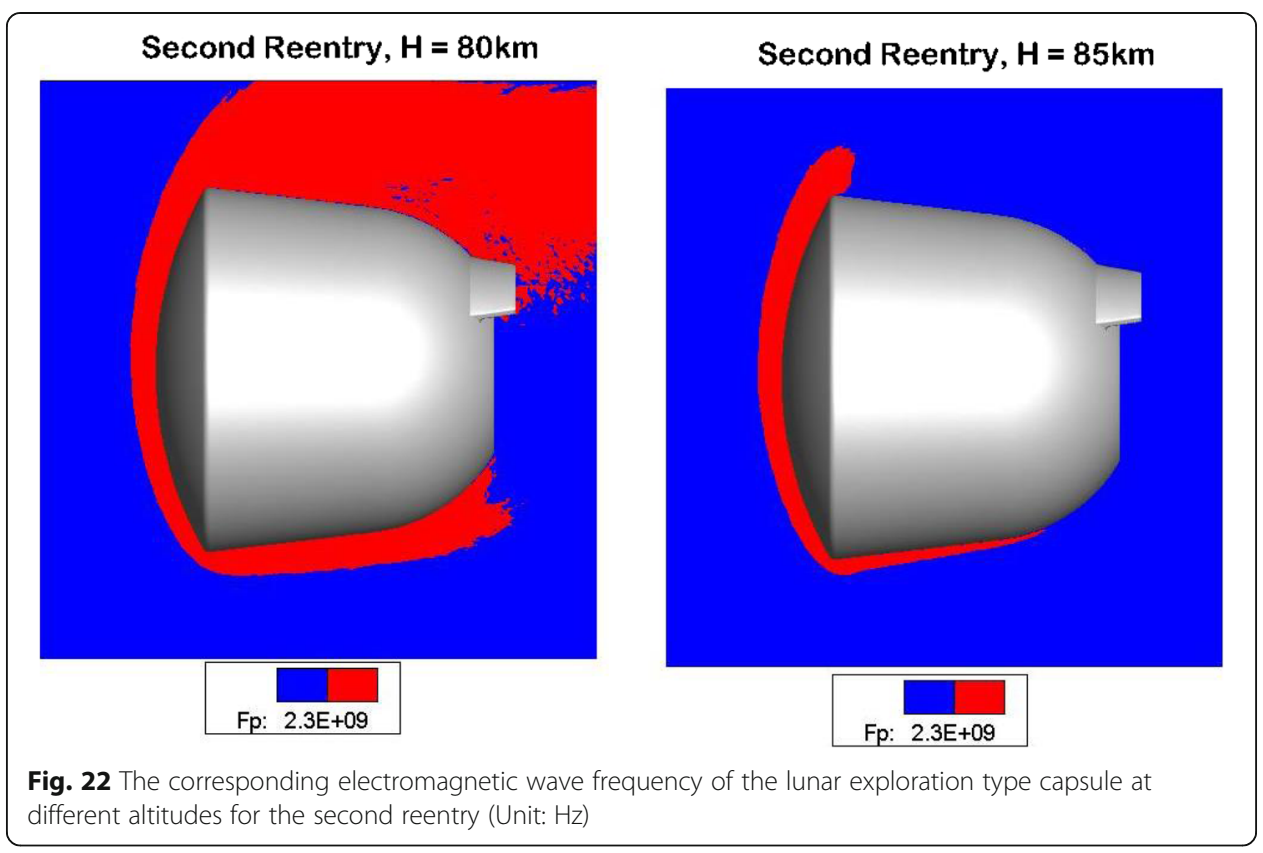

of trace species has been developed, which enables 3D simulations of rarefied air ionization with complex configurations of spacecraft. The present algorithm has been used to compute the weak rarefied atmospheric ionization of the RAM-C II vehicle, the moderate ionization of the Chinese lunar capsule, and the strong ionization of the Stardust at the flying altitude ranging from $80 \mathrm{~km}$ to $97 \mathrm{~km}$.

By comparing the flow field electron number density with those obtained by the flight measurement experiments at different probe locations, it is shown that the present DSMC results are in excellent agreement with the flight measured data. In comparison with the previous axisymmetric simulations for hypervelocity reentry flow at a zero angle of attack, the present DSMC algorithm can predict the distributions of the electron better. In addition, the present method is also able to simulate the flows over complex geometry of spacecraft at varying angles of attack. For that reason, it is also applied to analyze rarefied ionization for the reentry flow of the Chinese lunar capsule at the flying altitudes between 80 and $97 \mathrm{~km}$. In the rarefied transitional flow regime, the ionization reaction is found to cover the windward capsule surface and leads to communication blackout, which is confirmed by the actual reentry flight test data.

The introduction of rare species weighting factor scheme can significantly improve the smoothness of rare species number density contours, especially for that of electron in weak ionization case, while it has negligible effect on the macroscopic flow variables. From the present DSMC computations of the reentry flows of the RAM-C II, the

Table 9 Comparisons of complete communication blackout between predictions and the flight observations

\begin{tabular}{llll}
\hline State & Forecast value $(\mathrm{km})$ & Observed value $(\mathrm{km})$ & Difference $(\mathrm{km})$ \\
\hline First reentry & $95 \sim 97$ & 97.1 & $\sim 2$ \\
First jump-out & $\sim 85$ & 86.3 & $<2$ \\
Second reentry & $\sim 85$ & 83.5 & $<2$ \\
\hline
\end{tabular}


Stardust, and the Chinese lunar capsule, we find that ionization is extremely weak for a spacecraft reentry at the first cosmic velocity, while it is significant to cause communication blackout for a spacecraft reentry with the second cosmic speed, an important designing issue to prevent communication interruption. For example, the maximum electron number density is only about $0.01 \%$ of the molecular number density for the RAM-C II with $\mathrm{V}_{\infty}=7800 \mathrm{~m} / \mathrm{s}$, while it can be up to $3 \%$ for the Stardust reentry at an extremely high speed of $\mathrm{V}_{\infty}=12.8 \mathrm{~km} / \mathrm{s}$.

As this work is only the beginning of numerical forecast for reentry communication interruption of the thermochemical ionization reaction, further investigations on the hypervelocity reentry flows around recyclable spacecraft with real gas effects involving internal energy excitation, thermochemical ionization radiation non-equilibrium reactions, need to be studied in the future.

\title{
Acknowledgements
}

The corresponding author would like to thank Profs. Tom Scanlon, Xiao-Jun Gu, Jun Zhang and Lei Wu in University of Strathclyde, and his projects' group including Bo-Qiang Du, Ao-Ping Peng, Jun-Lin Wu, Xin-Yu Jiang, Lei-Ning Dang, Zhi-Yong Bai, Xu-Guo Li etc. for their valuable discussion and help, specially commemorate Prof. Jason M. Reese in University of Edinburgh during the corresponding author's visit period under funding of the Distinguished Visiting Fellowship from the UK Royal Academy of Engineering from Feb.1 to May 31,2016.

\section{Authors' contributions}

MF developed the DSMC method for the hypersonic reentry flows with internal energy excitation, thermochemical ionization non-equilibrium reactions. ZL designed the DSMC modeling of hypersonic reentry thermochemical nonequilibrium flows and the gas-kinetic unified algorithm for computable modeling of Boltzmann equation. ZL developed the DSMC algorithm for hypersonic reentry thermochemical non-equilibrium flows. JL designed the DSMC approaches for the hypervelocity reentry non-equilibrium flows and rarefied reentry aerodynamic characteristics around spacecraft. $Y Z$ analyzed and interpreted the reentry communication interruption during hypervelocity reentering into the atmosphere. All authors read and approved the final manuscript.

\section{Funding}

This work is supported by the National Natural Science Foundation (Nos. 11602288 \& 91530319), the National Key Basic Research and Development Program (2014CB744100), the National Outstanding Youth Fund (11325212) of China, and the UK Royal Academy of Engineering for Distinguished Visiting Fellowships (DVF1516/3/57).

\section{Availability of data and materials}

Data supporting the results reported in the article can be found including where generated during the study. By data, the minimal dataset that would be necessary to interpret, replicate and build upon the findings reported in the article. The datasets generated and/or analyzed during the current study are not publicly available due to the Chinese manned space engineering but are available from the corresponding author on reasonable request.

\section{Competing interests}

The authors declare that they have no competing interests.

\author{
Author details \\ ${ }^{1}$ Hypervelocity Aerodynamics Institute, China Aerodynamics Research and Development Center, P.O.Box 211, Mianyang \\ 621000, China. ${ }^{2}$ National Laboratory for Computational Fluid Dynamics, BUAA, No.37 Xueyuan Road, Beijing 100191, \\ China. ${ }^{3}$ James Weir Fluids Laboratory, Department of Mechanical \& Aerospace Engineering, University of Strathclyde, \\ Glasgow G1 1XJ, UK.
}

Received: 24 September 2019 Accepted: 29 December 2019

Published online: 17 March 2020

\section{References}

1. Sarma GSR (2000) Physico-chemical modelling in hypersonic flow simulation. Prog Aerosp Sci 36:281-349

2. Votta R, Schettino A, Bonfiglioli A (2013) Hypersonic high altitude aerothermodynamics of a space re-entry vehicle. Aerosp Sci Technol 25:253-265

3. Ozawa T, Zhong J, Levin DA, Boger D, Wright M (2007) Modeling of the Stardust reentry flows with ionization in DSMC. AIAA paper 2007-611

4. Boyd ID, Trumble K, Wright MJ (2007) Nonequilibrium particle and continuum analyses of Stardust entry for nearcontinuum conditions. AlAA paper 2007-4543

5. Fang M, Li ZH, Li ZH, Li CX (2018) DSMC approach for rarefied air ionization during spacecraft reentry. Commun Comput Phys 23(4):1167-1190

6. Takahashi Y, Nakasato R, Oshima N (2016) Analysis of radio frequency blackout for a blunt-body capsule in atmospheric reentry missions. Aerospace 3:1-19 
7. Hultmark M, Marusic I, Mckeon BJ, Morrison JF (2016) Introduction to topical issue on extreme flows. Exp Fluids 57(1):1-2

8. Scalabrin LC, Boyd ID (2006) Numerical simulation of weakly ionized hypersonic flow for reentry configurations. AIAA paper 2006-3773

9. Hash D, Gnoffo P, Barnhardt M, Candler G et al (2007) Fire Il calculations for hypersonic nonequilibrium aerothermodynamics code verification: DPLR, LAURA and US3D. AIAA paper 2007-605

10. Tsai CY, Chue R, Nicholson C, Tyll J (2009) Hypervelocity capability of HyPulse Shock Tunnel for radiative heat transfer measurements at lunar reentries. AlAA paper 2009-1516

11. Yang JY, Huang JC (1995) Rarefied flow computations using nonlinear model Boltzmann equations. J Comput Phys 120: 323-339

12. Titarev V, Dumbser M, Utyuzhnikov S (2014) Construction and comparison of parallel implicit kinetic solvers in three spatial dimensions. J Comput Phys 256:17-33

13. Rogozin OA, Aristov W, Peng AP, Li ZH (2018) Regularization and modeling of the Boltzmann collisional operator: Tcheremissine and Shakhov approaches. Proc. of $31^{\text {st }}$ Intern. Symposium on Rarefied Gas Dynamics, Glasgow, 23-27 July

14. Ho MT, Zhu LH, Wu L, Wang P, Guo ZL, Li ZH, Zhang YH (2019) A multi-level parallel solver for rarefied gas flows in porous media. Comput Phys Commun 234:14-25

15. Wang P, Wu L, Ho MT, Li J, Li ZH, Zhang YH (2020) The kinetic Shakhov-Enskog model for non-equilibrium flow of dense gases. J Fluid Mech 883:A48

16. Mieussens $L$ (2000) Discrete-velocity models and numerical schemes for the Boltzmann-BGK equation in plane and axisymmetric geometries. J Comput Phys 162(2):429-466

17. Morris AB, Varghese PL, Goldstein DB (2011) Monte Carlo solution of the Boltzmann equation via a discrete velocity model. J Comput Phys 230:1265-1280

18. Brull S, Mieussens L (2014) Local discrete velocity grids for deterministic rarefied flow simulations. J Comput Phys 266:22-46

19. Wu L, White C, Scanlon TJ, Reese JM, Zhang YH (2015) A kinetic model of the Boltzmann equation for nonvibrating polyatomic gases. J Fluid Mech 763:24-50

20. Meng JP, Zhang YH, Reese JM (2015) Numerical simulation of rarefied gas flows with specific heat flux boundary conditions. Commun Comput Phys 17:1185-1200

21. Rovenskaya O, Croce G (2015) Numerical investigation of the effect of boundary conditions for a highly rarefied gas flow using the GPU accelerated Boltzmann solver. Comput Fluids 110:77-87

22. Yang LM, Shu C, Wu J, Wang Y (2016) Numerical simulation of flows from free molecular regime to continuum regime by a DVM with streaming and collision processes. J Comput Phys 306:291-310

23. Li ZH, Zhang HX (2004) Study on gas kinetic unified algorithm for flows from rarefied transition to continuum. J Comput Phys 193:708-738

24. Li ZH, Zhang HX (2009) Gas-kinetic numerical studies of three-dimensional complex flows on spacecraft re-entry. J Comput Phys 228:1116-1138

25. Li ZH, Peng AP, Zhang HX, Yang JY (2015) Rarefied gas flow simulations using high-order gas-kinetic unified algorithms for Boltzmann model equations. Prog Aerosp Sci 74:81-113

26. Peng AP, Li ZH, Wu JL, Jiang XY (2016) Implicit gas-kinetic unified algorithm based on multi-block docking grid for multi-body reentry flows covering all flow regimes. J Comput Phys 327:919-942

27. Li ZH, Peng AP, Ma Q, Dang LN, Tang XW, Sun XZ (2019) Gas-kinetic unified algorithm for computable modeling of Boltzmann equation and application to aerothermodynamics for falling disintegration of uncontrolled Tiangong-No.1 spacecraft. Adv Aerodynamics 1:4. https://doi.org/10.1186/s42774-019-0009-4

28. Kolobov VI, Arslanbekov RR, Aristov W et al (2007) Unified solver for rarefied and continuum flows with adaptive mesh and algorithm refinement. J Comput Phys 223:589-608

29. Kolobov VI, Arslanbekov RR (2012) Towards adaptive kinetic-fluid simulations of weakly ionized plasmas. J Comput Phys Spec Issue Comput Plasma Phys 231:839-869

30. Xu K, Huang JC (2010) A unified gas-kinetic scheme for continuum and rarefied flows. J Comput Phys 229:7747-7764

31. Zhu LH, Guo ZL, Xu K (2016) Discrete unified gas kinetic scheme on unstructured meshes. Comput Fluids 127:211-225

32. Bird GA (1994) Molecular gas dynamics and the direct simulation of gas flows. Clarendon Press, Oxford

33. Liang J, Li ZH, Li XG, Shi WB (2018) Monte Carlo simulation of spacecraft reentry aerothermodynamics and analysis for ablating disintegration. Commun Comput Phys 23(4):1037-1051

34. Goldsworthy MJ, Macrossan MN, Abdel-Jawad MM (2007) Multiple reactions and trace species in the DSMC macroscopic chemistry method. Phys Fluids 19:116102

35. Bird GA (1987) Nonequilibrium radiation during re-entry at 10km/s. AlAA paper $87-1543$

36. Carlson AB, Hassan HA (1992) Direct simulation of re-entry flows with ionization. J Thermophys Heat Transf 6:400-404

37. Boyd ID (2007) Modeling of associative ionization reactions in hypersonic rarefied flows. Center for Turbulence Research, Annual Research Briefs.

38. Ozawa T, Wang A, Levin DA, Modest M (2008) Particle photon Monte Carlo method for simulating atomic radiation in hypersonic reentry flows in DSMC. AIAA paper 2008-3916

39. Boyd ID (2007) Modeling of plasma formation in rarefied hypersonic entry flows. AIAA Paper 2007-206

40. Fan J, Zhang YH, Jiang JZ (2014) Monte Carlo modeling of electron density in hypersonic rarefied gas flows. 29th International Symposium on Rarefied Gas Dynamics

41. Shevyrin AA, Vashchenkov PV, Bondar YA, Ivanov MS (2014) Validation of DSMC results for chemically nonequilibrium air flows against measurements of the electron number density in RAM-C II flight experiment. 29th International Symposium on Rarefied Gas Dynamics

42. Bondar Y, Shevyrin A, Ivanov M, Kashkovsky A (2011) DSMC modeling of high temperature chemical reactions in air. AIAA paper 2011-3128

43. Bird GA (2011) The Q-K model for gas-phase chemical reaction rates. Phys Fluids 23:106101

44. Boyd ID (2007) Modeling of associative ionization reactions in hypersonic rarefied flows. Phys Fluids 19:096102 
45. Li ZH, Fang M, Jiang XY, Wu JL (2013) Convergence proof of the DSMC method and the gas-kinetic unified algorithm for the Boltzmann equation. Sci China Phys Mech Astron 56(2):404-417

46. Li ZH, Fang M, Tang SQ (2013) Analysis of statistical noise in DSMC method. ACTA Aerodynamics Sinica 31(1):1-8

47. Gupta RN, Yos JM, Thompson RA, Lee KP (1990) A review of reaction rates and thermodynamic and transport properties for an 11-species air model for chermical and thermal nonequilibrium calculations to 30000K. NASA RP-1232.

48. Gallis MA, Bond RB, Torczynski JR (2010) Assessment of reaction-rate predictions of a collision-energy approach for chemical reactions in atmospheric flows. AlAA paper 2010-4499

49. Timothy J. Bartel (2003) Modelling neutral \& plasma chemistry with DSMC. Rarefied Gas Dynamics: 23rd International Symposium, edited by A.D. Ketsdever \& E.P. Muntz.

50. Boyd ID (1996) Conservative species weighting scheme for the direct simulation Monte Carlo method. J Thermophys Heat Transf 10:579-585

51. Bird GA (1989) Perception of numerical method in rarefied gas dynamics. AIAA paper 89-211

52. Fang M, Li ZH, Li ZH, Tian Y (2017) Three dimensional DSMC simulation and validation of rarefied air ionization process for sphere-cone blunt body reentry. Acta Aerodynamica Sinica 35(1):1-7

53. Li ZH, Zhang HX, Song F, Zeng S (2005) A gas kinetic algorithm for flows in microchannel. Int J Nonlinear Sci Numerical Simul 6(3):261-270

54. Sone Y, Takata S, Ohwada T (1990) Numerical analysis of the plane Couette flow of a rarefied gas in the basis of the linearized Boltzmann equation for hard-sphere molecules. Eur J Mech B/Fluids 9:273-288

55. Jones WL, Cross AE (1972) Electrostatic probe measurements of plasma parameters for two reentry flight experiments at 25000 feet per second. NASA TN D6617. N.Y.

56. Gupta RN, Li KP, Thompson RA, Yos JM (1991) Calculations and curve fits of thermodynamic and transport properties for equilibrium air to 30000K. NASA RP-1260

57. Heald MA, Wharton CB (1978) Plasma diagnostics with microwaves. Robert E. Krieger Publishing Company, London

\section{Publisher's Note}

Springer Nature remains neutral with regard to jurisdictional claims in published maps and institutional affiliations.

\section{Submit your manuscript to a SpringerOpen ${ }^{\circ}$ journal and benefit from:}

- Convenient online submission

- Rigorous peer review

- Open access: articles freely available online

- High visibility within the field

- Retaining the copyright to your article

Submit your next manuscript at $>$ springeropen.com 